\title{
Control of parameter dependent systems
}

\author{
Martin Lazar* Jérôme Lohéac ${ }^{\dagger}$
}

December 2, 2020

\begin{abstract}
In this chapter we provide an overview of recent progress on the problem of controllability of parameter dependent systems. We explore a different control notions successfully developed through the last decade. The aim of the control function is to steer the system to a state satisfying some properties prescribed either at some time instant $T>0$ or during a given time interval. These properties may be separated with respect to parameter values and can refer just to a single system itself (e.g. greedy control), or may consider solutions corresponding to the whole parameter range (e.g. ensemble control, averaged control). In the latter case control functions are designed as parameter invariant, implying a same control is to be applied to the system independently of a particular realisation of the parameter, while in the first case controls vary along with the parameter.

Beside the positive theoretical results, for each notion we provide a precise computational algorithm accompanied by a numerical example.
\end{abstract}

Keywords: Averaged control, greedy control, ensemble control, parameter dependent systems, reduced basis.

\section{Contents}

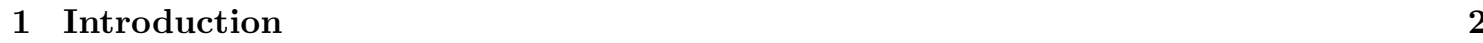

2 Parameter invariant controls 4

$2.1 \quad$ Averaged controllability . . . . . . . . . . . . . . . . . . . . . . . . . . . . . . . . . . . . . .

2.2 Ensemble controllability . . . . . . . . . . . . . . . . . . . . . . . 13

\begin{tabular}{|lll}
\hline & Parameter dependent controls & 17
\end{tabular}

3.1 Selection of a reduced basis . . . . . . . . . . . . . . . . . . . . . . . . . . . . . . . . . . . . . . . . .

3.2 The online procedure . . . . . . . . . . . . . . . . . . . . . . . . . 24

3.3 Other approaches . . . . . . . . . . . . . . . . . . . . . . 26

\begin{tabular}{|lr}
4 Conclusion & 28
\end{tabular}

*Department of Electrical Engineering and Computing, University of Dubrovnik, Ćira Carića 4, 20000 Dubrovnik, Croatia (mlazar@unidu.hr).

${ }^{\dagger}$ Université de Lorraine, CNRS, CRAN, F-54000 Nancy, France (jerome.loheac@univ-lorraine.fr). 


\section{Introduction}

In this chapter, we consider parameter dependent control systems of the form,

$$
\begin{aligned}
\dot{x}_{\theta}(t) & =A_{\theta} x_{\theta}(t)+B_{\theta} u_{\theta}(t) \quad(t>0), \\
x_{\theta}(0) & =\mathrm{x}_{\theta}^{0},
\end{aligned}
$$

where $x_{\theta}(t) \in \mathbb{R}^{N}$ is the state of the system, $u_{\theta}(t)$ is the control, and $\mathrm{x}_{\theta}^{0} \in \mathbb{R}^{N}$ is a given initial condition. The dynamics operator $A_{\theta}$ is an $N \times N$ matrix, the control operator $B_{\theta}$ is an $N \times M$ matrix, while $\theta$ is a parameter describing the system.

If not precised otherwise, the following assumptions will be used throughout the chapter.

\section{Assumption 1.}

(i) $\theta$ belongs to a compact set $\Theta \subset \mathbb{R}^{d}$;

(ii) $\theta \in \Theta \mapsto\left(A_{\theta}, B_{\theta}, \mathrm{x}_{\theta}^{0}\right) \in \mathbb{R}^{N \times N} \times \mathbb{R}^{N \times M} \times \mathbb{R}^{N}$ is Lipschitz continuous.

The aim of the control function is to steer the system to a state satisfying some properties prescribed either at some time instant $T>0$ or during a given time interval. These properties may be separated with respect to parameter values and can refer just to a single system itself (e.g. greedy control), or may consider solutions corresponding to the whole parameter range (e.g. ensemble control, averaged control). In the latter case controls $u_{\theta}$ are designed as parameter invariant, implying a same control is to be applied to the system (1.1) independently of a particular realisation of the parameter $\theta$, while in the first case the controls $u_{\theta}$ vary with $\theta$.

The Chapter is organised as follows. In the next subsection we present a brief introduction to controllability of finite dimensional systems. Section 2 deals with various types of parameter independent controls, and in Section 3, we present different methods for efficient design of (an approximation of) control $u_{\theta}$ for every parameter $\theta$. Each presented control notion is accompanied by a precise algorithm, providing construction of an optimal control function, and a numerical example.

Recall on finite dimensional control systems. Here we present some classical results on control of a general finite dimensional system of a form as in (1.1), with dependence with respect to parameter being omitted. A more elaborated overview of the topic can be found in e.g. 48, Chapter 1] or [52].

A system

$$
\dot{x}(t)=A x(t)+B u(t), \quad x(0)=\mathrm{x}^{0},
$$

is said to be controllable if every initial datum $\mathrm{x}^{0}$ can be driven to any final datum $\mathrm{x}^{1} \in \mathbb{R}^{N}$ in some (and consequently in any) time $T>0$.

This controllability property can be fully characterised by the so called Kalman rank condition. It says that system 1.2 is controllable if and only if

$$
\operatorname{rank}\left[B, A B, \ldots, A^{N-1} B\right]=N .
$$

This condition is of purely algebraic nature, and also time independent. For this reason controllability in some time $T>0$ implies controllability in any positive time, and it is easy to observe that there are infinitely many controls steering the trajectory from the initial datum to the prescribed final target. 
Another characterisation of the controllability property is provided by the dual problem of observability of the adjoint system

$$
\left\{\begin{array}{l}
-\dot{\varphi}=A^{*} \varphi \\
\varphi(T)=\varphi^{1}
\end{array} \quad(t \in(0, T))\right.
$$

More precisely the following result holds.

Proposition 1.1. System $(1.2)$ is controllable in time $T$ if and only if the adjoint system $(1.4)$ is observable in time $T$, i.e., if there exists a constant $C>0$ such that, for all solution $\varphi$ of (1.4),

$$
\left|\varphi^{1}\right|^{2} \leq C \int_{0}^{T}\left|B^{*} \varphi\right|^{2} \mathrm{~d} t .
$$

Both properties hold in all time $T>0$ if and only if the Kalman rank condition (1.3) is satisfied.

Furthermore, this dual approach provides a control of minimal $L^{2}$-norm out of all admissible controls. More precisely, if $\widetilde{\varphi}^{1}$ is a minimiser of the quadratic functional $J: \mathbb{R}^{N} \rightarrow \mathbb{R}$ :

$$
J\left(\varphi^{1}\right)=\frac{1}{2} \int_{0}^{T}\left|B^{*} \varphi(t)\right|^{2} \mathrm{~d} t-\left\langle\mathrm{x}^{1}, \varphi^{1}\right\rangle+\left\langle\mathrm{x}^{0}, \varphi(0)\right\rangle,
$$

then the control

$$
u=B^{*} \widetilde{\varphi} \in L^{2}(0, T)^{M},
$$

where $\widetilde{\varphi}$ is the corresponding solution of 1.4 , is the control of minimal $L^{2}$-norm steering the system from $\mathrm{x}^{0}$ to $\mathrm{x}^{1}$ in time $T$.

Note that the minimiser $\widetilde{\varphi}^{1}$ of the functional $J$ exists since the latter is differentiable, convex and, by the observability inequality (1.5), coercive. In addition, it satisfies the requirement $D J\left(\widetilde{\varphi}^{1}\right)=0$ which can be equivalently restated as

$$
\Lambda \widetilde{\varphi}^{1}=\mathrm{x}^{1}-e^{T A} \mathrm{x}^{0} .
$$

Here the operator $\Lambda$ stands for the (infinite time) controllability Gramian associated to the pair $(A, B)$, given by the relation

$$
\Lambda=\int_{0}^{T} e^{(T-t) A} B B^{*} e^{(T-t) A^{*}} \mathrm{~d} t .
$$

Thus solving the control problem reduces to finding the minimiser of the functional (1.6), which is equivalent to solving the system (1.7). In practice, this is done by exploring (conjugate) gradient or some other iterative method. Note this is usually implemented without construction of the Gramian matrix $\Lambda$ itself. Indeed, application of the Gramian operator to any vector $\varphi^{1}$ corresponds to the state of the system at time $T$ driven by the control of the form $u=B^{*} \varphi$, where $\varphi$ is the solution of the adjoint system (1.4). Thus, each iteration requires solving in turn the dual and the primal system, which for systems of a large dimension $N$ represents a significant computational challenge, specially if it has to be resolved and applied in a real time.

For these reasons, when dealing with large systems that are in addition parameter dependent, it is of crucial importance to develop robust algorithms that provide (at least approximatively) desired control functions in a stable and computationally efficient way. 


\section{Parameter invariant controls}

In this paragraph, we are looking for controls $u_{\theta}$ independent of the parameter $\theta$, that is to say that we consider the system

$$
\begin{aligned}
\dot{x}_{\theta}(t) & =A_{\theta} x_{\theta}(t)+B_{\theta} u(t) \quad(t>0), \\
x_{\theta}(0) & =\mathrm{x}_{\theta}^{0} .
\end{aligned}
$$

More precisely, in order to define the controllability notion, we propose two different approaches. In the first notion, (averaged controllability), the goal is to steer the expectation of the system to the target, while for the second one (ensemble controllability), the aim is to steer every realisation of the system to an arbitrarily small ball around the target. Of course for the averaged controllability notion, it is required that the parameter realisation follows some probability law. One can see that ensemble controllability is a stronger notion, and it has been checked in [4] that ensemble controllability implies averaged controllability.

In each case, the major goal is to steer the parameter dependent solution to some target by a single control. The problem is relevant in applications in which the control has to be chosen in a robust manner, independently of a particular realisation of parameters obeying some deterministic or stochastic law.

Let us make more precise these two notions. To this end, we denote by $x_{\theta}\left(t ; x_{\theta}^{0}, u\right)$ the solution of (2.1) at time $t$.

Definition 1 (Averaged controllability). Assume that Assumption 2 holds, and let $(\Theta, \mathcal{F}, \mu)$ be a probability space (i.e., $\mathcal{F}$ is a $\alpha$-algebra on $\Theta$ and $\mu$ is a probability measure defined on $\mathcal{F}$ ). We say that the system (2.1) is controllable in average, with respect to the probability distribution $\mu$, if for every $\mu$-integrable function $\theta \in \Theta \mapsto \mathrm{x}_{\theta}^{0} \in \mathbb{R}^{N}$, and every $\overline{\mathrm{x}}^{1} \in \mathbb{R}^{N}$, there exist a time $T>0$ and a control $u \in L^{2}(0, T)^{M}$ such that the solution of (2.1) satisfies

$$
\int_{\Theta} x_{\theta}\left(T ; \mathrm{x}_{\theta}^{0}, u\right) \mathrm{d} \mu_{\theta}=\overline{\mathrm{x}}^{1} .
$$

Of course, for Definition 1 to make sense, one need an integrability property of $\theta \mapsto x_{\theta}\left(T ; \mathrm{x}_{\theta}^{0}, u\right)$. This can be ensured using Assumption2 2 Note however that more general well-posedness conditions can be stated in terms of the adjoint state (see [53]).

Assumption 2 (Averaged controllability assumptions). $\theta \in \Theta \mapsto\left(A_{\theta}, B_{\theta}\right) \in \mathbb{R}^{N \times N} \times \mathbb{R}^{N \times M}$ is $\mu$-measurable, and $\sup \left\{\operatorname{Re}\left(\lambda_{\theta}\right) \mid \lambda_{\theta} \in \sigma\left(A_{\theta}\right), \theta \in \Theta\right\}<\infty$.

Hence, for averaged controllability, i.e., in Section 2.1. only Assumption 2 will be assumed.

Definition 2 (Ensemble controllability). Assume that Assumption 1 holds. We say that the system (2.1) is ensemble controllable, if for every $\varepsilon>0$, every continuous function $\theta \in \Theta \mapsto\left(\mathrm{x}_{\theta}^{0}, \mathrm{x}_{\theta}^{1}\right) \epsilon$ $\mathbb{R}^{N} \times \mathbb{R}^{N}$, there exist a time $T>0$ and a control $u \in L^{2}(0, T)^{M}$ such that the solution of (2.1) satisfies

$$
\sup _{\theta \in \Theta}\left|x_{\theta}\left(T ; \mathrm{x}_{\theta}^{0}, u\right)-x_{\theta}^{1}\right|<\varepsilon .
$$

Remark 2.1. - By taking the formal limit $\varepsilon \rightarrow 0$ in Definition 2, we obtain the notion of simultaneous controllability (see e.q. 44] or [36, Chapter 5] for this notion). However, let us mention that if the cardinal of $\Theta$ is infinite, then simultaneous controllability cannot hold, see [47. In addition, when $\Theta=\left\{\theta_{1}, \ldots, \theta_{K}\right\}$, then the ensemble controllability of 2.1] 
coincide with the simultaneous controllability of (2.1), that is to say to the controllability of the pair $(\mathbb{A}, \mathbb{B})$, with

$$
\mathbb{A}=\left(\begin{array}{cccc}
A_{\theta_{1}} & 0 & \cdots & 0 \\
0 & \ddots & \ddots & \vdots \\
\vdots & & \ddots & 0 \\
0 & \cdots & 0 & A_{\theta_{K}}
\end{array}\right) \in \mathbb{R}^{K N \times K N} \quad \text { and } \quad \mathbb{B}=\left(\begin{array}{c}
B_{\theta_{1}} \\
\vdots \\
\vdots \\
B_{\theta_{K}}
\end{array}\right) \in \mathbb{R}^{K N \times M}
$$

This is the consequence of the well-known result stating that approximate controllability implies exact controllability in finite dimension.

- Let us mention that Definition 2 is also called uniform ensemble controllability. Indeed, one can consider the more general notion of $L^{q}(\Theta, \mu)$-ensemble controllability, for $q \in(1, \infty)$ and some measure $\mu$ on $\Theta$, by substituting 2.3 with $\left(\int_{\Theta}\left|x_{\theta}\left(T ; \mathrm{x}_{\theta}^{0}, u\right)-x_{\theta}^{1}\right|^{q} \mathrm{~d} \mu_{\theta}\right)^{1 / q}<\varepsilon$. Note however that for a bounded measure $\mu$, the ensemble controllability defined by Definition 2 implies $L^{q}(\Theta, \mu)$-ensemble controllability. This is an easy consequence of the continuous injection of $C^{0}(\Theta) \cap L^{\infty}(\Theta)$ in $L^{\infty}(\Theta ; \mu)$ and of $L^{\infty}(\Theta ; \mu)$ in $L^{q}(\Theta ; \mu)$.

In the rest of this section, we consider averaged controllability in Section 2.1 and ensemble controllability in Section 2.2. For each of these notions, we recall some known results and propose numerical methods to compute a control realisation.

\subsection{Averaged controllability}

\subsubsection{Averaged controllability notion and results}

The notion of averaged controllability has first been introduced in 53. Rapidly averaged controllability results has been obtained for different classes of PDE systems, see e.g. [4, 5, 12, 25, 27, 30, 38, 39, 41. Links between averaged and simultaneous controllability has been obtained in 37. and a refined version of averaged controllability (the notion of long time averaged controllability has been performed in [28]). Dealing with numerical aspects of averaged controllability, we refer to 1 f for wave equations and to 41 for Euler-Bernoulli plate equations.

In [53, it has been shown that the system (2.1) is controllable in average if and only if a Kalman type rank condition is satisfied. It has also been shown there, that the averaged controllability property is equivalent to the positivity of some controllability Gramian. In addition, if the system (2.1) is controllable in average, then one can build a control of minimal $L^{2}$-norm. These results are summarized in the following theorem.

Theorem 2.2 ([53]). The following properties are equivalent.

1. The system (2.1) is controllable in average, with probability measure $\mu$.

2. $\operatorname{rank}\left[\int_{\Theta} B_{\theta} \mathrm{d} \mu_{\theta}, \int_{\Theta} A_{\theta} B_{\theta} \mathrm{d} \mu_{\theta}, \ldots, \int_{\Theta} A_{\theta}^{k} B_{\theta} \mathrm{d} \mu_{\theta}, \ldots\right]=N$.

3. There exists $T>0$ such that the $N \times N$ matrix

$$
\bar{\Lambda}(T)=\int_{0}^{T}\left(\int_{\Theta} e^{(T-t) A_{\theta}} B_{\theta} \mathrm{d} \mu_{\theta} \int_{\Theta} B_{\theta}^{*} e^{(T-t) A_{\theta}^{*}} \mathrm{~d} \mu_{\theta}\right) \mathrm{d} t
$$

is positive, i.e., there exist a constant $c(T)>0$ such that $\varphi^{1^{*}} \bar{\Lambda}(T) \varphi^{1} \geqslant c(T)\left|\varphi^{1}\right|$ for every $\varphi^{1} \in \mathbb{R}^{N}$.

4. For every $T>0$, the matrix $\bar{\Lambda}(T)$, defined by (2.4), is positive. 
In addition, if the system (2.1) is controllable in average, then for every $\mu$-integrable $\left(\mathrm{x}_{\theta}^{0}\right)_{\theta \in \theta} \epsilon$ $\left(\mathbb{R}^{N}\right)^{\Theta}$, every $\overline{\mathrm{x}}^{1} \in \mathbb{R}^{N}$, and every $T>0$, then there exist a unique control of minimal $L^{2}$-norm steering the initial condition $\left(\mathrm{x}_{\theta}^{0}\right)_{\theta}$ to the averaged value $\overline{\mathrm{x}}^{1}$ in time $T$, and this control is given by

$$
u(t)=\int_{\Theta} B_{\theta}^{*} e^{(T-t) A_{\theta}^{*}} \bar{\Lambda}(T)^{-1}\left(\overline{\mathrm{x}}^{1}-\int_{\Theta} e^{T A_{\theta}} \mathrm{x}_{\theta}^{0} \mathrm{~d} \mu_{\theta}\right) \mathrm{d} \mu_{\theta} \quad(t \in(0, T)) .
$$

Remark 2.3. Note that the $3{ }^{\mathrm{d}}$ condition of Theorem 2.2 is also equivalent to the observability of the adjoint. More precisely, this condition is equivalent to,

$$
c(T)\left|\varphi^{1}\right|^{2} \leqslant \int_{0}^{T}\left|\int_{\Theta} B_{\theta}^{*} \varphi_{\theta}(t) \mathrm{d} \mu_{\theta}\right|^{2} \mathrm{~d} t \quad\left(\varphi^{1} \in \mathbb{R}^{N}\right),
$$

where $\varphi_{\theta}$ is solution of

$$
\begin{aligned}
-\dot{\varphi}_{\theta}(t) & =A_{\theta}^{*} \varphi_{\theta}(t) \quad(t \in(0, T)), \\
\varphi_{\theta}(T) & =\varphi^{1} .
\end{aligned}
$$

Note also that we trivially have the existence of a constant $C(T)>0$ such that

$$
\varphi^{1^{*}} \bar{\Lambda}(T) \varphi^{1} \leqslant C(T)\left|\varphi^{1}\right|^{2} \quad\left(\varphi^{1} \in \mathbb{R}^{N}\right),
$$

Remark 2.4. Note that the control of minimal $L^{2}$-norm given in Theorem 2.2 is given by

$$
u(t)=\int_{\Theta} B_{\theta}^{*} \varphi_{\theta}(t) \mathrm{d} \mu_{\theta},
$$

where $\varphi_{\theta}$ is solution of 2.7 , with $\varphi^{1} \in \mathbb{R}^{N}$ solution of the minimization problem,

$$
\begin{array}{cl}
\min & \frac{1}{2} \int_{0}^{T}\left|\int_{\Theta} B_{\theta}^{*} \varphi_{\theta}(t) \mathrm{d} \mu_{\theta}\right|^{2} \mathrm{~d} t-\left\langle\varphi^{1}, \overline{\mathrm{x}}^{1}\right\rangle+\int_{\Theta}\left\langle\varphi_{\theta}(0), \mathrm{x}_{\theta}^{0}\right\rangle \mathrm{d} \mu_{\theta} \\
& \left.\varphi^{1} \in \mathbb{R}^{N} \text { and } \varphi_{\theta} \text { solution of } 2.7\right) .
\end{array}
$$

This expression of the control is easier to consider, since we only have to solve a quadratic minimization problem in $\mathbb{R}^{N}$ to obtain $\varphi^{1}$. The associated minimal $L^{2}$-norm control is then obtained from (2.9). More precisely, in order to solve the quadratic problem, one can use for instance any gradient descent method. Then, once $\varphi^{1}$ has been found, we obtain $\varphi_{\theta}(t)$ by solving (2.7), and finally, using the expression (2.9), we get $u(t)$. Of course, in this procedure, one has to discretize time and integrals with respect to $\Theta$. Note that the time discretization is rather standard and hence will not be developed here. The discretization with respect to $\theta$, will be discussed in the rest of this section.

\subsubsection{Numerical approximation}

In this subsection we deal with the issue of constructing a (numerical approximation of) averaged control for a general system for which formulae 2.5) or (2.9) can not be calculated exactly, either due to computational complexity or to issues related to integration (with respect to $\theta$ ).

The idea behind all the numerical methods presented here is to consider $K \in \mathbb{N}^{*}$ values $\theta_{1}^{K}, \ldots, \theta_{K}^{K} \in \Theta$ and $K$ weights $m_{1}^{K}, \ldots, m_{K}^{K} \in \mathbb{R}_{+}$, so that $\sum_{k=1}^{K} m_{k}^{K} e^{t A_{\theta_{k}^{K}}} B_{\theta_{k}^{K}}$ converges for every $t \in[0, T]$ to $\int_{\Theta} e^{t A_{\theta}} B_{\theta} \mathrm{d} \mu_{\theta}$, as $K$ goes to $\infty$. Once these parameters are given, we consider the 
matrices,

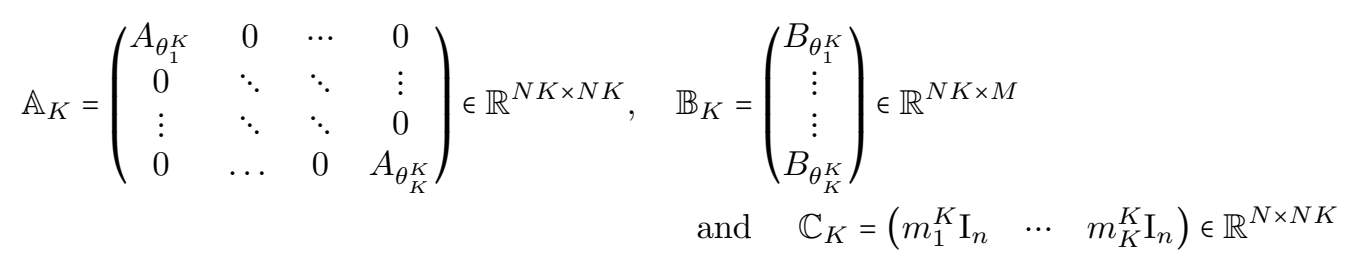

and the system

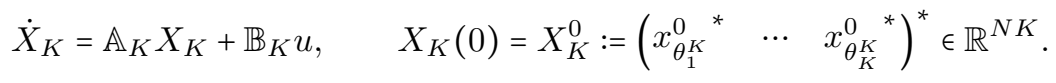

with output

$$
\bar{x}_{K}=\mathbb{C}_{K} X_{K} .
$$

Given some time $T>0$, the problem is to find a control $u \in L^{2}(0, T)^{M}$ such that the output of the system (2.11) satisfies $\bar{x}_{K}(T)=\overline{\mathrm{x}}^{1}$.

Definition 3. We say that the system (2.11) is output controllable if for every $X^{0} \in \mathbb{R}^{N K}$ and every $\overline{\mathrm{x}}^{1} \in \mathbb{R}^{N}$, there exist a time $T>0$ and a control $u \in L^{2}(0, T)^{M}$ such that the solution of (2.11) satisfies, $\bar{x}_{K}(T)=\overline{\mathrm{x}}^{1}$.

Remark 2.5. The notion of output controllability is more general than the averaged one, and can be applied for general systems $\dot{x}=A x+B u$, with output $y=C x+D u$. We refer to [26] for this notion and corresponding controllability results.

Remark 2.6. Note that the output controllability of 2.11) coincides with the averaged controllability of 2.1 with the measure $\mu_{K}=\sum_{k=1}^{N} m_{k}^{K} \delta_{\theta_{k}^{K}}$, where $\delta_{\theta}$ is the Dirac measure supported at $\theta \in \Theta$. As a consequence output controllability criteria for the system (2.11) can be directly obtained from Theorem 2.2. In particular, (2.11) is averaged controllable if and only if for every $T>0$, the Gramian

$$
\bar{\Lambda}_{K}(T)=\mathbb{C}_{K}\left(\int_{0}^{T} e^{(T-t) \mathbb{A}_{K}} \mathbb{B}_{K} \mathbb{B}_{K}^{*} e^{(T-t) \mathbb{A}_{K}^{*}} \mathrm{~d} t\right) \mathbb{C}_{K}^{*}
$$

is positive. In addition, if $\bar{\Lambda}_{K}(T)$ is positive, the minimal $L^{2}$-norm control in time $T>0$ is given by

$$
u_{K}(T)=\mathbb{B}_{K}^{*} e^{(T-t) \mathbb{A}_{K}^{*}} \mathbb{C}_{K}^{*} \bar{\Lambda}_{K}(T)^{-1}\left(\overline{\mathrm{x}}^{1}-\mathbb{C}_{K} e^{T \mathbb{A}_{K}} X_{K}^{0}\right) .
$$

Algorithm 1 will be then used to compute numerically an averaged control for $K$ evaluations of the system.

In practice, given some error $\varepsilon>0$, one wants to determine as integer $K=K(\varepsilon)$ such that $\left\|u_{K}-u\right\|_{L^{2}(0, T)^{M}} \leqslant \varepsilon$ (which in turn implies that $\left|\bar{x}\left(T ; \mathrm{x}_{\theta}^{0}, u_{K}\right)-\mathrm{x}_{\theta}^{1}\right|$ is small as well), where $u$ is the control of minimal $L^{2}$-norm such that $\bar{x}_{\theta}\left(T ; \mathrm{x}_{\theta}^{0}, u\right)=\overline{\mathrm{x}}^{1}$. The difficulty is that, in practice, we know the convergence rate, but do not know the multiplicative constant in front of it, i.e., with the notations of Proposition 2.8 (eq. (2.14)), we know $p_{o}$, but not $C$. To overcome this difficulty, one check a posteriori that $\left|\bar{x}\left(T ; \mathrm{x}_{\theta}^{0}, u_{K}\right)-\mathrm{x}_{\theta}^{1}\right|<\varepsilon$.

Remark 2.7. Obviously, to compute $\left|\bar{x}\left(T ; \mathrm{x}_{\theta}^{0}, u_{K}\right)-\bar{x}_{\theta}^{1}\right|$ in Algorithm 2, one needs again to discretized $\int_{\theta} x_{\theta}\left(T ; \mathrm{x}_{0}^{\theta}, u_{K}\right) \mathrm{d} \mu_{\theta}$, this can be done with a finer discretization $\theta_{1}, \ldots, \theta_{L}$ than the one $\left(\theta_{1}^{K}, \ldots, \theta_{K}^{K}\right)$ used in Algorithm 1. 

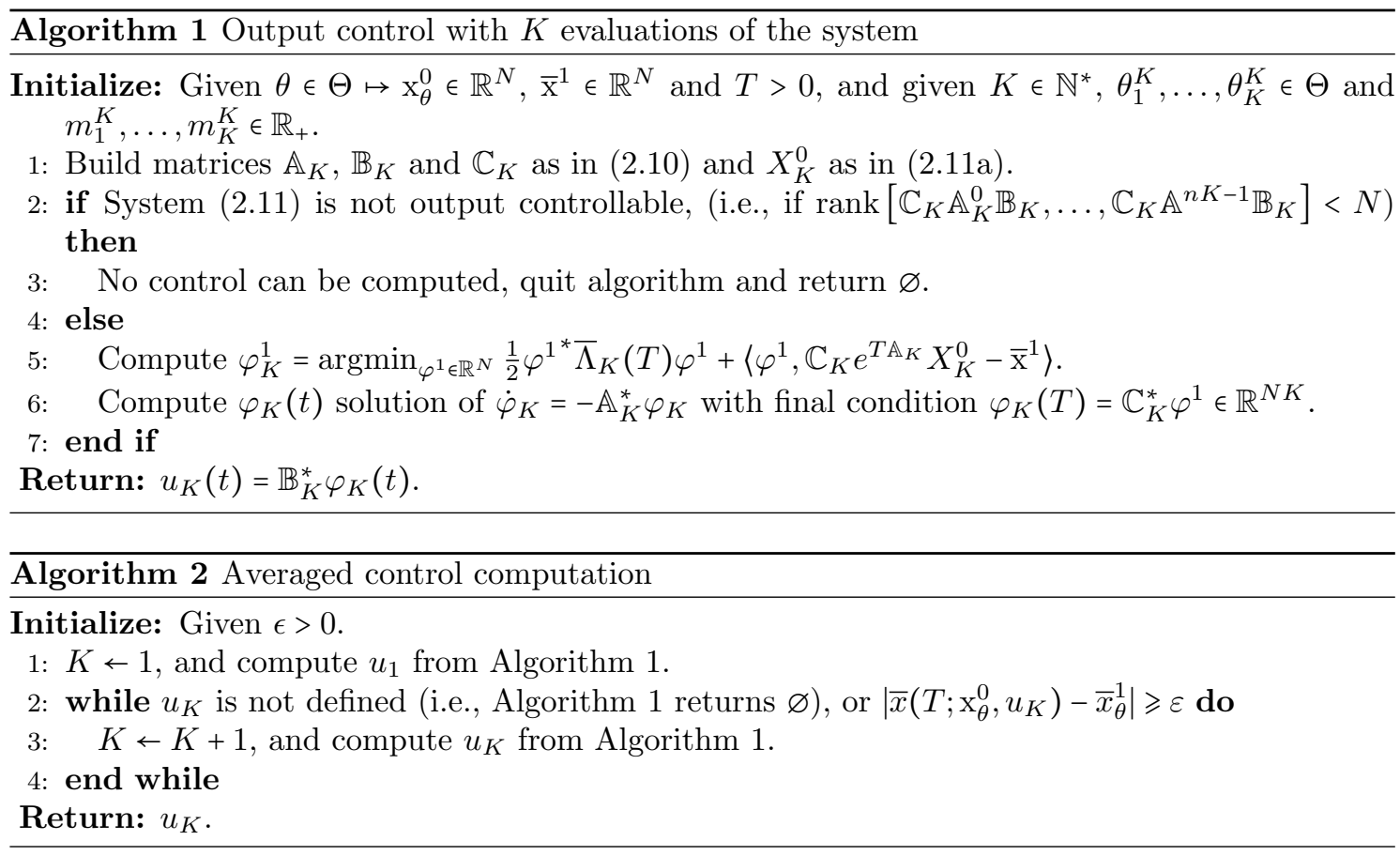

Based on the expressions given in Remark 2.6 and with well-chosen parameters $\theta_{1}^{K}, \ldots, \theta_{K}^{K}$ and $m_{1}^{K}, \ldots, m_{K}^{K}$, one can ensure that if $K$ is large enough, then the system (2.11) is output controllable. In addition, the numerical control $u_{K}$ given by Algorithm 1 converges to the exact control as $K \rightarrow \infty$.

Proposition 2.8. Let $T>0, \overline{\mathrm{x}}^{1} \in \mathbb{R}^{N}$ and assume that 2 2.1) is $\mu$-averaged controllable. Assume in addition that $\theta_{1}^{K}, \ldots, \theta_{K}^{K} \in \Theta$, and $m_{1}^{K}, \ldots, m_{K}^{K} \in \mathbb{R}_{+}$are designed so that there exist $C_{o}>0$ and $p_{o}>0$ such that

$$
\begin{aligned}
\sup _{t \in[0, T]}\left|\int_{\Theta} e^{t A_{\theta}} B_{\theta} \mathrm{d} \mu_{\theta}-\sum_{k=1}^{K} m_{k}^{K} e^{t A_{\theta_{k}^{K}}} B_{\theta_{k}^{K}}\right| & \leqslant C_{o} K^{-p_{o}} \\
& \text { and }\left|\int_{\Theta} e^{T A_{\theta}} \mathrm{x}_{\theta}^{0} \mathrm{~d} \mu_{\theta}-\sum_{k=1}^{K} m_{k}^{K} e^{T A_{\theta_{k}^{K}}} \mathrm{x}_{\theta_{k}^{K}}^{0}\right| \leqslant C_{o} K^{-p_{o}} .
\end{aligned}
$$

Then for $K \in \mathbb{N}^{*}$ large enough, the system 2.11) is output controllable.

Furthermore, there exist $K_{0} \in \mathbb{N}^{*}$ and $C>0$ such that

$$
\left\|u_{K}-u\right\|_{L^{2}(0, T)^{M}} \leqslant C K^{-p_{o}} \quad \text { and } \quad\left|\int_{\Theta} x_{\theta}\left(T ; \mathrm{x}_{\theta}^{0}, u_{K}\right) \mathrm{d} \mu_{\theta}-\overline{\mathrm{x}}^{1}\right| \leqslant C K^{-p_{o}} \quad\left(K \geqslant K_{0}\right),
$$

where $u$ and $u_{K}$ are the controls respectively given by (2.5) and (2.13), and where $x_{\theta}\left(\cdot ; \mathrm{x}_{\theta}^{0}, u_{K}\right)$ is the solution of 2.1a with control $u_{K}$.

Proof. Let us first proof the existence of $K_{0} \in \mathbb{N}$ such that for every $K \geqslant K_{0}$, the system (2.11) is output controllable. To this end, we define the integration error

$$
E_{K}(t)=\sum_{k=1}^{K} m_{k}^{K} e^{t A_{\theta_{k}^{K}}} B_{\theta_{k}^{K}}-\int_{\Theta} e^{t A_{\theta}} B_{\theta} \mathrm{d} \mu_{\theta} \quad(t \in[0, T]) .
$$


We observe that $\bar{\Lambda}_{K}(T)$ given by 2.12 can be expressed as

$$
\begin{array}{r}
\bar{\Lambda}_{K}(T)=\bar{\Lambda}(T)+\int_{0}^{T}\left(\int_{\Theta} e^{t A_{\theta}} B_{\theta} \mathrm{d} \mu_{\theta}\right) E_{K}(t)^{*} \mathrm{~d} t+\int_{0}^{T} E_{K}(t)\left(\int_{\Theta} e^{t A_{\theta}} B_{\theta} \mathrm{d} \mu_{\theta}\right)^{*} \mathrm{~d} t \\
+\int_{0}^{T} E_{K}(t) E_{K}(t)^{*} \mathrm{~d} t .
\end{array}
$$

Hence, for every $\varphi^{1} \in \mathbb{R}^{N}$, we get,

$$
\varphi^{1^{*}} \bar{\Lambda}_{K}(T) \varphi^{1} \geqslant \varphi^{1^{*}} \bar{\Lambda}(T) \varphi^{1}-2 \sqrt{T C(T)}\left|\varphi^{1}\right|^{2} C_{o} K^{-p_{o}}-T C_{0}^{2} K^{-2 p_{o}}\left|\varphi^{1}\right|^{2} .
$$

with $C(T)$ given by Remark 2.3. Since the system 2.1) is averaged controllable, we have $\varphi^{1 *} \bar{\Lambda}(T) \varphi^{1} \geqslant c(T)\left|\varphi^{1}\right|^{2}$ (here again, $c(T)$ is defined by Remark 2.3). This ensures the existence of $K_{0} \in \mathbb{N}^{*}$ such that for every $K \geqslant K_{0}$, we have,

$$
\varphi^{1^{*}} \bar{\Lambda}_{K}(T) \varphi^{1} \geqslant c_{K}(T)\left|\varphi^{1}\right|^{2} \quad\left(\varphi^{1} \in \mathbb{R}^{N}\right),
$$

for some positive constant $c_{K}(T)$. That is to say that the system 2.11$)$ is output controllable.

Proving the convergence rate of $u_{K}$ to $u$ is a classical exercise which is not developed here. This fact also leads to the convergence of $\int_{\Theta} x_{\theta}\left(T, u_{K}\right) \mathrm{d} \mu_{\theta}$ to $\overline{\mathrm{x}}^{1}$.

In the rest of this section, we check this result in Example 2.12 with two different interpolations rules. The first one is based on the mid-point rule, while the second one on the large number theory.

Interpolation approach. For this approach, we assume that the set of parameter $\Theta$ is some real interval. We will also assume ${ }^{1}$ that $\mathrm{d} \mu_{\theta}=w(\theta) \mathrm{d} \theta$, where $w$ is some positive function on $\Theta$. Let us then define for every $\theta \in \Theta, W(\theta)=\mu(\Theta \cap(-\infty, \theta))=\int_{\Theta \cap(-\infty, \theta)} w(s) \mathrm{d} s$. It is clear that $W$ is an increasing function, and hence, $W: \Theta \rightarrow[0,1]$ is a bijective map.

In order to apply Proposition 2.8, one need to have a consistent numerical method for the integration with respect to the measure $\mu$. This can be for instance done with the mid-point rule. To this end, we define for every $K \in \mathbb{N}^{*}, K+1$ points $z_{0}^{K}, \ldots, z_{K}^{K} \in[0,1]$ satisfying $0=z_{0}^{K}<z_{1}^{K}<$ $\cdots<z_{K-1}^{K}<z_{K}^{K}=1$, and set

$$
\theta_{k}^{K}=W^{-1}\left(\frac{z_{k-1}^{K}+z_{k}^{K}}{2}\right) \quad \text { and } \quad m_{k}^{K}=z_{k}^{K}-z_{k-1}^{K} \quad(k \in\{1, \ldots, K\})
$$

and for every $f \in C^{0}(\Theta)$, such that $\int_{\Theta}|f| \mathrm{d} \mu<\infty$, we numerically approach $\int_{\Theta} f \mathrm{~d} \mu$ by

$$
I_{K}(f)=\sum_{k=1}^{K} m_{k}^{K} f\left(\theta_{k}^{K}\right) .
$$

Lemma 2.9. In addition to the above notations and assumptions, let us assume that $f$ and $W$ are $C^{2}$ functions on $\Theta$. Let us also set $g: \Theta \rightarrow \mathbb{R}_{+}$a $\mu$-integrable function such that $g \geqslant|f|$. Then we have

$$
\left|\int_{\Theta} f(\theta) \mathrm{d} \mu_{\theta}-\sum_{k=1}^{K} m_{k}^{K} f\left(\theta_{k}^{K}\right)\right| \leqslant \sum_{k=2}^{K-1} \frac{M_{k}}{24}\left(m_{k}^{K}\right)^{3}+r_{1}+r_{K} .
$$

\footnotetext{
${ }^{1}$ The following description can also be adapted for more general probability measures (including the one having a countable number of atomic masses). However, we keep this assumption for the sake of simplicity.
} 
where we have set

$$
\begin{aligned}
& M_{k}=\sup _{\left[W^{-1}\left(z_{k-1}^{K}\right), W^{-1}\left(z_{k}^{K}\right)\right]} w^{-2}\left|f^{\prime \prime}-f^{\prime} \frac{w^{\prime}}{w}\right| \quad(k \in\{2, \ldots, K-1\}), \\
& r_{1}=\left\{\begin{array}{ll}
\sup _{\substack{\left.\inf \Theta, W^{-1}\left(z_{1}^{K}\right)\right] \\
z_{1}^{K}}} w^{-2}\left|f^{\prime \prime}-f^{\prime} \frac{w^{\prime}}{w}\right| \frac{\left(m_{1}^{K}\right)^{3}}{24} & \text { if } \lim _{z \rightarrow 0} f \circ W^{-1}(z) \text { exists, } \\
\int_{0}^{z^{K}} g \circ W^{-1} \mathrm{~d} z+m_{1}^{K} g\left(\theta_{1}^{K}\right) & \text { otherwise }
\end{array}\right. \text { and } \\
& r_{K}= \begin{cases}\sup _{\left[W^{-1}\left(z_{K-1}^{K}\right), \sup \Theta\right)} w^{-2}\left|f^{\prime \prime}-f^{\prime} \frac{w^{\prime}}{w}\right| \frac{\left(m_{K}^{K}\right)^{3}}{24} & \text { if } \lim _{z \rightarrow 1} f \circ W^{-1}(z) \text { exists } \\
\int_{z_{K-1}^{K}}^{1} g \circ W^{-1} \mathrm{~d} z+m_{K}^{K} g\left(\theta_{K}^{K}\right) & \text { otherwise. }\end{cases}
\end{aligned}
$$

Proof. Let us first observe that,

$$
\int_{\Theta} f(\theta) \mathrm{d} \mu_{\theta}=\int_{\Theta} f(\theta) w(\theta) \mathrm{d} \theta=\int_{0}^{1} f\left(W^{-1}(z)\right) \mathrm{d} z=\sum_{k=1}^{K} \int_{z_{k-1}^{K}}^{z_{k}^{K}} f\left(W^{-1}(z)\right) \mathrm{d} z .
$$

Note that $f \circ W^{-1}$ is of class $C^{2}$ on $(0,1)$ but might not be defined at 0 or 1 . Hence, for $k \in$ $\{2, \ldots, K-1\}$, we classically have

$$
\left|\int_{z_{k-1}^{K}}^{z_{k}^{K}} f \circ W^{-1} \mathrm{~d} z-m_{k}^{K} f\left(\theta_{k}^{K}\right)\right| \leqslant \frac{\left(z_{k}^{K}-z_{k-1}^{K}\right)^{3}}{24} \sup _{\left[z_{k-1}^{K}, z_{k}^{K}\right]}\left|\frac{\mathrm{d}^{2} f \circ W^{-1}}{\mathrm{~d} z^{2}}\right| .
$$

We observe that $\frac{\mathrm{d}^{2} f \circ W^{-1}}{\mathrm{~d} z^{2}} \circ W=\frac{1}{w^{2}}\left(f^{\prime \prime}-f^{\prime} \frac{w^{\prime}}{w}\right)$ and hence, we have,

$$
\left|\int_{z_{k-1}^{K}}^{z_{k}^{K}} f \circ W^{-1} \mathrm{~d} z-m_{k}^{K} f\left(\theta_{k}^{K}\right)\right| \leqslant M_{k}\left(z_{k}^{K}-z_{k-1}^{K}\right)^{3} .
$$

Note that this estimate is also valid for $k=1$ (respectively $k=K$ ) if $f \circ W^{-1}$ is continuous (or admits a continuous extension) at 0 (respectively 1$)$. When this does not hold, we can however use the following estimate, for $k=1$ or $K=K$,

$$
\left|\int_{z_{k-1}^{K}}^{z_{k}^{K}} f \circ W^{-1} \mathrm{~d} z-m_{k}^{K} f\left(\theta_{k}^{K}\right)\right| \leqslant \int_{z_{k-1}^{K}}^{z_{k}^{K}} g \circ W^{-1} \mathrm{~d} z+m_{k}^{K} g\left(\theta_{k}^{K}\right) \quad(k \in\{1, \ldots, K\}) .
$$

Randomized approach. For this approach, we consider $K \in \mathbb{N}^{*}$ realisations of the random variable $\theta$, following the probability law $\mu$. This leads to some parameters $\theta_{1}^{K}, \ldots, \theta_{K}^{K} \in \Theta$. We then apply the output controllability procedure to the system 2.11), with matrices $\mathbb{A}, \mathbb{B}$ and $\mathbb{C}$ given by 2.10), with $m_{k}^{K}=1 / K$.

More precisely, we approach $I(f)=\int_{\Theta} f(\theta) \mathrm{d} \mu_{\theta}$ by the random variable

$$
I_{K}(f)=\frac{1}{K} \sum_{k=1}^{K} f\left(\theta_{k}^{K}\right)
$$

where $\theta_{k}^{K}$ is a random variable following the probability law $\mu$. Based on the central limit Theorem, we know that the expectation of the error, $I(f)-I_{K}(f)$ is null, and furthermore, $\sqrt{K}(I(f)-$ $\left.I_{K}(f)\right) / \sigma$ converges in law to a random variable following the probability law $\mathcal{N}(0,1)$, where $\sigma$ is the standard derivation of $f(\theta)$. More precisely, we have the following result. 
Lemma 2.10. Given $(\Theta, \mathcal{F}, \mu)$ a probability space and let $f: \Theta \rightarrow \mathbb{R}$ be a measurable function such that $\int_{\Theta}|f(\theta)|^{2} \mathrm{~d} \mu_{\theta}<\infty$. We define $I(f)=\int_{\Theta} f(\theta) \mathrm{d} \mu_{\theta}$ and $\sigma=\sqrt{\int_{\Theta}(f(\theta)-I(f))^{2} \mathrm{~d} \mu_{\theta}}$ Then for every sequence $\left(\theta_{k}\right)_{k \in \mathbb{N}^{*}}$ of independent and identically distributed random variables following the probability law $\mu$, we have that $\sqrt{K}\left(I_{K}(f)-I(f)\right) / \sigma$ (with $I_{K}(f)$ defined by 2.18) converges in law to the normal distribution $\mathcal{N}(0,1)$.

Lemma 2.10 ensures in particular that for every $a>0$, the probability that $\left|I_{K}(f)-I(f)\right|<$ $a / \sqrt{K}$ converges to a constant as $K \rightarrow \infty$. That is to say that, when evaluating the random variable $\theta_{1}, \ldots, \theta_{K}$, it is expected that $I(f)-I_{K}(f)$ decays polynomially with rate $1 / 2$. This rate will be confirmed on Example 2.12 .

Remark 2.11. Note that this method is very simple to implement in practice. Its convergence rate is rather small, but it is easy to extend this method for general parameter set $\Theta$ and general measure $\mu$. In addition, the convergence rate of this method is independent of the size of $\Theta$.

Numerical example. To conclude this subsection, we consider both the interpolation and randomized approaches on an example.

Example 2.12. Let us consider the probability space $\left(\mathbb{R}_{+}, \mathcal{B}\left(\mathbb{R}_{+}\right), \mu\right)$, where the measure $\mu$ is given by $\mathrm{d} \mu_{\theta}=w(\theta) \mathrm{d} \theta$, with $w(\theta)=e^{-\theta}$. Let us also assume that the dimension of the state is $N=2$ and the dimension of the control is $M=1$, we then consider the matrices $A_{\theta}$ and $B_{\theta}$ given by

$$
A_{\theta}=\left(\begin{array}{cc}
0 & -\theta^{2} \\
1 & 0
\end{array}\right) \quad \text { and } \quad B_{\theta}=\left(\begin{array}{c}
0 \\
\theta-1
\end{array}\right) \quad(\theta \in \Theta) .
$$

For this example, everything can be computed by hands. In particular, for $T=1$, the controllability Gramian $\bar{\Lambda}(T)$ given in 2.4 is

$$
\bar{\Lambda}(1)=\frac{1}{240}\left(\begin{array}{cc}
32+15 \pi & 30 \\
30 & 15 \pi-20
\end{array}\right) .
$$

One can observe that $\bar{\Lambda}(1)$ is a positive matrix. Hence, the system consider in this example is controllable in average. In addition, we set as initial condition $\mathrm{x}_{\theta}^{0}=\left(\begin{array}{ll}\theta & \sin \theta\end{array}\right)^{*}$ and an averaged target condition $\overline{\mathrm{x}}^{1}=0$. Using the relation $(2.5)$, we obtain that the control steering $\left(\mathrm{x}_{\theta}^{0}\right)_{\theta}$ on the average $\overline{\mathrm{x}}^{1}$ in time $T=1$ is

$$
u_{e x}(t)=\frac{48(t-1)\left(144-90 \pi+520 t-156 \pi t-188 t^{2}+33 \pi t^{2}-72 t^{3}+45 \pi t^{3}\right)}{5\left(45 \pi^{2}+36 \pi-308\right)\left(2-2 t+t^{2}\right)^{3}} \quad(t \in[0,1]) .
$$

In order to apply Proposition 2.8 , we have to consider the $\mu$-integration of $f$, with $f$ given by $f(\theta)=e^{T A_{\theta}} \mathbf{x}_{\theta}^{0}$ or $f(\theta)=e^{t A_{\theta}} B_{\theta}$.

- Interpolation appraoch. Our aim is to apply Lemma 2.9 with well-chosen values $z_{k}^{K}$. First note that we have,

$$
w(\theta)^{-2}\left|f^{\prime \prime}(\theta)-f^{\prime}(\theta) \frac{w^{\prime}(\theta)}{w(\theta)}\right|=e^{2 \theta}\left|f^{\prime \prime}(\theta)+f^{\prime}(\theta)\right| \quad\left(\theta \in \mathbb{R}_{+}\right) .
$$

In order to estimate $f^{\prime}$ and $f^{\prime \prime}$, one can use the following procedure. For $f(\theta)=e^{T A_{\theta}} \mathrm{x}_{\theta}^{0}$, let us define for every $t \in[0, T], \xi_{\theta}(t)=e^{t A_{\theta}} \mathrm{x}_{\theta}^{0}$, so that $\xi_{\theta}(T)=f(\theta)$. Then, $\xi_{\theta}, \xi_{\theta}^{\prime}$ and $\xi_{\theta}^{\prime \prime}$ (where "'" denotes the differentiation with respect to $\theta$ ) are solution of

$$
\left(\begin{array}{c}
\dot{\xi}_{\theta} \\
\dot{\xi}_{\theta}^{\prime} \\
\dot{\xi}_{\theta}^{\prime \prime}
\end{array}\right)=\left(\begin{array}{ccc}
A_{\theta} & 0 & 0 \\
A_{\theta}^{\prime} & A_{\theta} & 0 \\
A_{\theta}^{\prime \prime} & 2 A_{\theta}^{\prime} & A_{\theta}
\end{array}\right)\left(\begin{array}{c}
\xi_{\theta} \\
\xi_{\theta}^{\prime} \\
\xi_{\theta}^{\prime \prime}
\end{array}\right), \quad \quad \quad\left(\begin{array}{c}
\xi_{\theta}(0) \\
\xi_{\theta}^{\prime}(0) \\
\xi_{\theta}^{\prime \prime}(0)
\end{array}\right)=\left(\begin{array}{c}
x_{\theta}^{0} \\
x_{\theta}^{0^{\prime}} \\
x_{\theta}^{0^{\prime \prime}}
\end{array}\right) .
$$


In our particular case, we get,

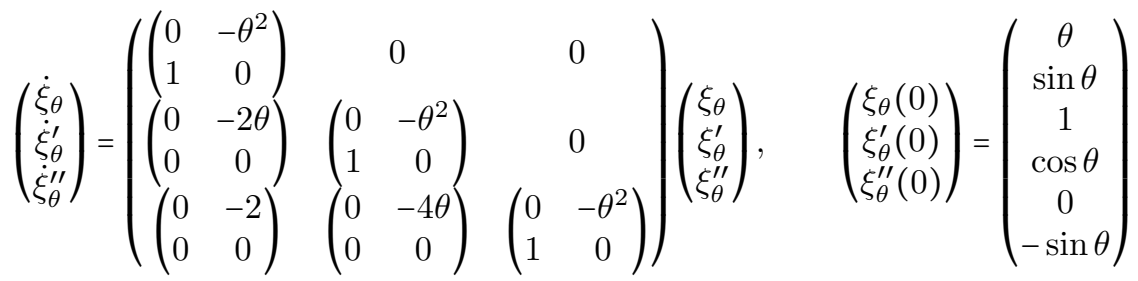

and easily obtain

$$
e^{2 \theta}\left|f^{\prime \prime}(\theta)+f^{\prime}(\theta)\right|=e^{2 \theta}\left|\xi_{\theta}^{\prime \prime}(T)+\xi_{\theta}^{\prime}(T)\right| \leqslant C_{\varepsilon} e^{(2+\varepsilon) \theta},
$$

for every $\varepsilon>0$ and for some constant $C_{\varepsilon}>0$ (indeed $\left|\xi_{\theta}(T)\right|^{2}+\left|\xi_{\theta}^{\prime}(T)\right|^{2}+\left|\xi_{\theta}^{\prime \prime}(T)\right|$ is polynomial in $(T, \theta)$ and hence can be bounded by an exponential). In addition, since the eigenvalues of $A_{\theta}$ are purely complex numbers, we have,

$$
|f(\theta)|=\left|\mathrm{x}_{\theta}^{0}\right| \leqslant \theta+1 \text {. }
$$

Similarly, for $f(\theta)=e^{t A_{\theta}} B_{\theta}$, we consider, $\varphi^{1} \in \mathbb{R}^{N}$ and $\varphi_{\theta}$ solution of $-\dot{\varphi}_{\theta}=A_{\theta}^{*} \varphi_{\theta}$ with $\varphi_{\theta}(T)=\varphi^{1}$, we then have that,

$$
-\left(\begin{array}{c}
\dot{\varphi}_{\theta} \\
\dot{\varphi}_{\theta}^{\prime} \\
\dot{\varphi}_{\theta}^{\prime \prime}
\end{array}\right)=\left(\begin{array}{ccc}
A_{\theta}^{*} & 0 & 0 \\
A_{\theta}^{\prime *} & A_{\theta}^{*} & 0 \\
A_{\theta}^{\prime \prime *} & 2 A_{\theta}^{\prime *} & A_{\theta}^{*}
\end{array}\right), \quad \quad \quad\left(\begin{array}{c}
\varphi_{\theta}(0) \\
\varphi_{\theta}^{\prime}(0) \\
\varphi_{\theta}^{\prime \prime}(0)
\end{array}\right)=\left(\begin{array}{c}
\varphi^{1} \\
0 \\
0
\end{array}\right)
$$

and that,

$$
\begin{aligned}
\left(f^{\prime \prime}+f^{\prime}\right)^{*} \varphi^{1}=B_{\theta}^{\prime \prime *} \varphi_{\theta}(t)+B_{\theta}^{\prime *}\left(2 \varphi_{\theta}^{\prime}(t)+\varphi_{\theta}(t)\right)+ & B_{\theta}^{*}\left(\varphi_{\theta}^{\prime \prime}(t)+\varphi_{\theta}^{\prime}(t)\right) \\
& =\mathrm{e}_{2}^{*}\left((\theta-1) \varphi_{\theta}^{\prime \prime}(t)+(\theta+1) \varphi_{\theta}^{\prime}(t)+\varphi_{\theta}(t)\right),
\end{aligned}
$$

with $\mathrm{e}_{2}^{*}=\left(\begin{array}{ll}0 & 1\end{array}\right)$. Here also, we get that,

$$
e^{2 \theta}\left|\left(f^{\prime \prime}(\theta)+f^{\prime}(\theta)\right)^{*} \varphi^{1}\right|=e^{2 \theta}\left|(\theta-1) \varphi_{\theta}^{\prime \prime}(t)+(\theta+1) \varphi_{\theta}^{\prime}(t)+\varphi_{\theta}(t)\right| \leqslant C_{\varepsilon} e^{(2+\varepsilon) \theta}\left|\varphi^{1}\right|,
$$

for every $\varepsilon>0$, every $\varphi^{1} \in \mathbb{R}^{2}$ and for some constant $C_{\varepsilon}>0$ independant of $\varphi^{1}$ and $t$. In addition, we have,

$$
\left|f(\theta)^{*} \varphi^{1}\right|=\left|\varphi_{\theta}(t)\right|=\left|\varphi^{1}\right| \leqslant(\theta+1)\left|\varphi^{1}\right| \quad\left(\varphi^{1} \in \mathbb{R}^{2}, t \in[0, T]\right) .
$$

Finally, for both definition of $f$, we get from Lemma 2.9 (with $g(\theta)=\theta+1$ ), that the interpolation error $E_{f}^{K}=\left|\int_{\Theta} f(\theta) \mathrm{d} \mu_{\theta}-\sum_{k=1}^{K} m_{k}^{K} f\left(\theta_{k}^{K}\right)\right|$ satisfies,

$$
\begin{aligned}
E_{f}^{K} \leqslant C_{\varepsilon} \sum_{k=1}^{K-1} e^{(2+\varepsilon) W^{-1}\left(z_{k}^{K}\right)}\left(z_{k}^{K}-z_{k-1}^{K}\right)^{3}+\left(2+W^{-1}\left(z_{K-1}^{K}\right)\right) & e^{-W^{-1}\left(z_{K-1}^{K}\right)} \\
& +\left(1-z_{K-1}^{K}\right)\left(W^{-1}\left(\frac{z_{K-1}^{K}+1}{2}\right)+1\right) .
\end{aligned}
$$

Using the fact that $W^{-1}(z)=-\ln (1-z)$, and setting $z_{k}^{K}=k / K$ for every $k \in\{0, \ldots, K\}$, we finally get

$$
E_{f}^{K} \leqslant \frac{1}{K^{1-\varepsilon}}\left(C_{\varepsilon} \frac{2+\varepsilon}{1+\varepsilon}+\frac{3+2 \ln (2 K)}{K^{\varepsilon}}-\frac{C_{\varepsilon}}{(1+\varepsilon) K^{1+\varepsilon}}\right) .
$$

That is to say that the interpolation error decays polynomially with rate $1-\varepsilon$, and we can apply Proposition 2.8, with $C_{o}=C_{\varepsilon}$ and $p_{o}=1-\varepsilon$, to obtain that the control $u_{K}-u_{e x}$ decays polynomially with rate $1-\varepsilon$. This fact is confirmed on Figure 1 a 
- Randomized approach. As already noticed, in both cases we have $|f(\theta)| \leqslant \theta+1$. This ensures that $\int_{\Theta}|f(\theta)|^{2} \mathrm{~d} \mu_{\theta}$ is finite. Hence, according to Lemma 2.10 the randomized approach is convergent in expectation with rate $1 / 2$. And hence by application of Proposition 2.8 , we obtain that $u_{K}-u_{e x}$ decays polynomially in expectation with rate $1 / 2$. This fact is confirmed numerically on Figure $1 \mathrm{~b}$

Note that in order to generate the sequence of random variables, we observe that there is a one-to-one corresponance between random variable $\theta \in\left(\mathbb{R}_{+}, \mathcal{B}\left(\mathbb{R}_{+}\right), \mu\right)$ and random variable $z \epsilon$ $((0,1), \mathcal{B}((0,1)), \lambda)$ (with $\lambda$ the Lebesgue measure). More precisely, if $z \in((0,1), \mathcal{B}((0,1)), \lambda)$, then $\theta=W^{-1}(z) \in\left(\mathbb{R}_{+}, \mathcal{B}\left(\mathbb{R}_{+}\right), \mu\right)$, with $W(s)=\mu([0, s])$.

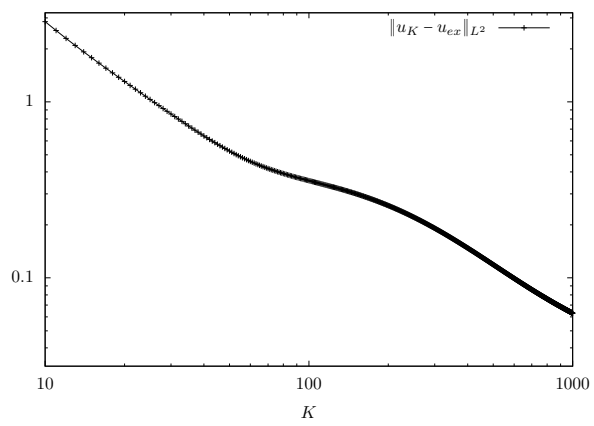

(a) For the interpolation approach, we numerically obtain polynomially decay rate equal to 0.761540 .

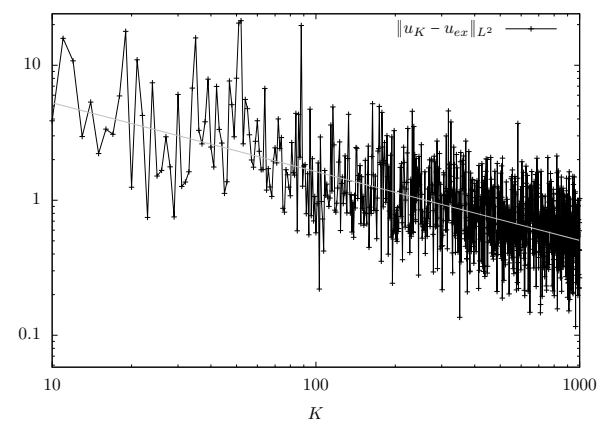

(b) For the randomised approach, we numerically obtain polynomially decay rate equal to 0.509645 .

Figure 1: Convergence as the number of evaluation points goes to $\infty$ for Example 2.12.

\subsection{Ensemble controllability}

\subsubsection{Ensemble controllability notion and results}

The notion of ensemble controllability has been at first stage introduced for Bolch equation, and we refer to [6, 33] for related articles. We also refer to [3, 11] for ensemble controllability for more general nonlinear systems. Dealing with linear control system, this notion has been studied in [14, 19, 32, 35, 34, 51].

A necessary and sufficient condition for ensemble controllability can be stated.

Proposition 2.13. ([47, Theorem 3.1.1] or [14, Section 2]) The system (2.1) is ensemble controllable if and only if $\operatorname{Span}\left\{\theta \in \Theta \mapsto A_{\theta}^{k} B_{\theta} \mathrm{e}_{j} \mid k \in \mathbb{N}, j \in\{1, \ldots, M\}\right\}$ is dense in $C^{0}(\Theta)^{N}$, where $\mathrm{e}_{1}, \ldots, \mathrm{e}_{M}$ denote vectors of the canonical basis of $\mathbb{R}^{M}$.

Note that if the system 2.1) is ensemble controllable, then it is ensemble controllable in any time $T>0$, see 14 .

The condition of Proposition 2.13 might be hard to check, and instead we shall employ the following sufficient condition.

\footnotetext{
${ }^{1}$ Strictly speaking, Proposition 2.8 cannot be used. Indeed, one shall rely on its randomized version, whose proof is similar to the original proof of Proposition 2.8
} 
Proposition 2.14. ([14, Corollary 3]) In addition to Assumption 1 , let us assume that $M=1$ (i.e., $B_{\theta} \in \mathbb{R}^{N}$ ). The system (2.1) is ensemble controllable if the following conditions are satisfied:

1. the pair $\left(A_{\theta}, B_{\theta}\right)$ is controllable for every $\theta \in \Theta$;

2. the eigenvalues of $A_{\theta}$ are simple for every $\theta \in \Theta$;

3. the map $\theta \in \Theta \mapsto \sigma\left(A_{\theta}\right) \in \mathcal{P}(\mathbb{C})$ is injective.

\subsubsection{Numerical approximation}

In this paragraph, we propose an algorithm to compute, for a given precision $\varepsilon>0$, given time $T>0$, and given initial and target datas $\theta \mapsto\left(\mathrm{x}_{\theta}^{0}, \mathrm{x}_{\theta}^{1}\right)$, a control $u^{\varepsilon}$ such that 2.3 holds. To this end, we first state a regularity lemma.

Lemma 2.15. Let us assume, in addition to Assumption 1 that $\theta \mapsto \mathrm{x}_{\theta}^{1}$ is Lipschitz continuous. Then, for every $T>0$ and every $u \in L^{2}(0, T)^{M}$, the map $\theta \in \Theta \mapsto \mathrm{x}_{\theta}^{1}-x_{\theta}\left(T ; \mathrm{x}_{\theta}^{0}, u\right) \in \mathbb{R}^{N}$ is Lipschitz continuous with Lipschitz constant $C_{0}+C_{1}\|u\|_{L^{2}(0, T)^{M}}$, where $C_{0}$ and $C_{1}$ are two nonnegative constants independent of $u$.

Proof. Recall, that we have, $x_{\theta}\left(T ; \mathrm{x}_{\theta}^{0}, u\right)=e^{T A_{\theta}} \mathrm{x}_{\theta}^{0}+\int_{0}^{T} e^{(T-t) A_{\theta}} B_{\theta} u(t) \mathrm{d} t$. The conclusion follows by noticing that $\theta \mapsto e^{t A_{\theta}}$ is Lipschitz continuous with a Lipschitz constant independent of $t \in$ $[0, T]$.

Let us also state a simple lemma.

Lemma 2.16. Assume that the system (2.1) is ensemble controllable, and let $\theta \in \Theta \mapsto\left(\mathrm{x}_{\theta}^{0}, \mathrm{x}_{\theta}^{1}\right) \in$ $\mathbb{R}^{N} \times \mathbb{R}^{N}$ be any continuous function on $\Theta$. Then for every $T>0$, every $\varepsilon>0$, every $K \geqslant 2$ and every set of distinct values $\theta_{1}, \ldots, \theta_{K} \in \Theta$, there exist a control $u_{K}^{\varepsilon} \in L^{2}(0, T)^{M}$ such that the solution $x_{\theta}\left(\cdot ; \mathrm{x}_{\theta}^{0}, u_{K}^{\varepsilon}\right)$ of 2.1 satisfies

$$
\sup _{k \in\{1, \ldots, K\}}\left|\mathrm{x}_{\theta_{k}}^{1}-x_{\theta_{k}}\left(T ; \mathrm{x}_{\theta_{k}}^{0}, u_{K}^{\varepsilon}\right)\right| \leqslant \varepsilon .
$$

Furthermore the control of minimal $L^{2}$-norm such that 2.21 holds, is given by

$$
u_{K}^{\varepsilon}(t)=\sum_{k=1}^{K} B_{\theta_{k}}^{*} \varphi_{k}(t)
$$

where we have set $\varphi_{k}(t)$ the solution of,

$$
-\dot{\varphi}_{k}=A_{\theta_{k}}^{*} \varphi_{k}, \quad \varphi_{k}(T)=\varphi_{k}^{1},
$$

with $\left(\varphi_{1}^{1}, \ldots, \varphi_{K}^{1}\right) \in\left(\mathbb{R}^{N}\right)^{K}$ the minimiser of

$$
J\left(\varphi_{1}^{1}, \ldots, \varphi_{K}^{1}\right)=\frac{1}{2} \int_{0}^{T}\left|\sum_{k=1}^{K} B_{\theta_{k}}^{*} e^{(T-t) A_{\theta_{k}}^{*}} \varphi_{k}^{1}\right|^{2} \mathrm{~d} t+\sum_{k=1}^{K}\left\langle\varphi_{k}^{1}, e^{T A_{\theta_{k}}} \mathrm{x}_{\theta_{k}}^{0}-\mathrm{x}_{\theta_{k}}^{1}\right\rangle+\varepsilon \sum_{k=1}^{K}\left|\varphi_{k}^{1}\right| .
$$

Proof. The existence of a control $u_{K}^{\varepsilon} \in L^{2}(0, T)^{M}$ such that 2.21 holds directly follows from Definition 2. We then deduce the existence of a unique minimal $L^{2}$-norm control such that (2.21) holds. This control minimizes, $u \in L^{2}(0, T)^{M} \mapsto f(u)+g(\Phi u) \in \mathbb{R}$, with

$$
\begin{gathered}
f(u)=\frac{1}{2}\|u\|_{L^{2}(0, T)^{M}}^{2}, \quad \Phi u=\left(\int_{0}^{T} e^{\left.(T-t) A_{\theta_{k}} B_{\theta_{k}} u(t) \mathrm{d} t\right)_{k \in\{1, \ldots, K\}}} \quad\left(u \in L^{2}(0, T)^{M}\right),\right. \\
g\left(\left(X_{k}\right)_{k \in\{1, \ldots, K\}}\right)=\left\{\begin{array}{ll}
+\infty & \text { if } \sup _{k \in\{1, \ldots, K\}} \mid \mathrm{x}_{\theta_{k}}^{1}-e^{T A_{\theta_{k}} \mathrm{x}_{\theta_{k}}^{0}-X_{k} \mid>\varepsilon,} \\
0 & \text { otherwise }
\end{array} \quad\left(X_{1}, \ldots, X_{K} \in \mathbb{R}^{N}\right) .\right.
\end{gathered}
$$


Using Fenchel-Rockafellar duality, see for instance [9, 16], we obtain that the minimiser $u_{K}^{\varepsilon}$ of $u \mapsto f(u)+g(\Phi u)$ is given by $u_{K}^{\varepsilon}=\nabla f^{*}\left(-\Phi^{*} P^{1}\right)$, where $P^{1}=\left(\varphi_{k}^{1}\right)_{k \in\{1, \ldots, K\}} \in\left(\mathbb{R}^{N}\right)^{K}$ minimizes $P \in\left(\mathbb{R}^{N}\right)^{K} \mapsto f^{*}\left(\Phi^{*} P\right)+g^{*}(P)$. In these expressions, $\Phi^{*}$ is the adjoint of $\Phi$ and $f^{\star}$ (respectively $g^{\star}$ ) is the convex conjugate of $f$ (respectively $g$ ). More precisely, we have,

$$
\left(\Phi^{*}\left(\varphi_{k}^{1}\right)_{k \in\{1, \ldots, K\}}\right)(t)=\sum_{k=1}^{K} B_{\theta_{k}}^{*} e^{(T-t) A_{\theta_{k}}^{*}} \varphi_{k}^{1},
$$

that is to say that $\Phi^{*}\left(\varphi_{k}^{1}\right)_{k \in\{1, \ldots, K\}}=\sum_{k=1}^{K} B_{\theta_{k}}^{*} \varphi_{k}$, with $\varphi_{k}$ defined by 2.23 . We also have,

$$
f^{\star}(\varphi)=\sup _{u \in L^{2}(0, T)^{M}}(\langle\varphi, u\rangle-f(u))=\frac{1}{2}\|\varphi\|_{L^{2}(0, T)^{M}}^{2}
$$

and

$$
\begin{aligned}
g^{\star}\left(\left(\varphi_{k}^{1}\right)_{k \in\{1, \ldots, K\}}\right)= & \sup _{X_{1}, \ldots, X_{k} \in \mathbb{R}^{N}}\left(\sum_{k=1}^{K}\left\langle\varphi_{k}^{1}, X_{k}\right\rangle-g\left(\left(X_{k}\right)_{k \in\{1, \ldots, K\}}\right)\right) \\
& =\sup _{\substack{Y_{1}, \ldots, Y_{k} \in \mathbb{R}^{N} \\
\left|Y_{1}\right|, \ldots, Y_{K} \mid \leqslant 1}} \sum_{k=1}^{K}\left\langle\varphi_{k}^{1}, \mathrm{x}_{\theta_{k}}^{1}-e^{T A_{\theta_{k}}} \mathrm{x}_{\theta_{k}}^{0}-\varepsilon Y_{k}\right\rangle=\sum_{k=1}^{K}\left\langle\varphi_{k}^{1}, \mathrm{x}_{\theta_{k}}^{1}-e^{T A_{\theta_{k}}} \mathrm{x}_{\theta_{k}}^{0}\right\rangle+\varepsilon \sum_{k=1}^{K}\left|\varphi_{k}^{1}\right| .
\end{aligned}
$$

All these expressions lead to the claim of Lemma 2.16 .

Using Lemmas 2.15 and 2.16 we can deduce the following result.

Proposition 2.17. Assume that the system (2.1) is ensemble controllable, and let $\theta \in \Theta \mapsto$ $\left(\mathrm{x}_{\theta}^{0}, \mathrm{x}_{\theta}^{1}\right) \in \mathbb{R}^{N} \times \mathbb{R}^{N}$ be any Lipschitz continuous function on $\Theta$. Then for every $T>0$, every $\varepsilon>0$, every $\varepsilon_{0} \in(0, \varepsilon)$, there exist $K \in \mathbb{N}^{*}$ such that the solution $x_{\theta}\left(\cdot ; \mathrm{x}_{\theta}^{0}, u_{K}^{\varepsilon_{0}}\right)$ of (2.1) satisfies

$$
\sup _{\theta \in \Theta}\left|\mathrm{x}_{\theta}^{1}-x_{\theta}\left(T ; \mathrm{x}_{\theta}^{0}, u_{K}^{\varepsilon_{0}}\right)\right| \leqslant \varepsilon
$$

where $u_{K}^{\varepsilon_{0}}$ is the control of minimal $L^{2}$-norm such that (2.21) holds with $\theta_{k}=\min \Theta+(\max \Theta-$ $\min \Theta) \frac{k-1}{K-1}$ for $k \in\{1, \ldots, K\}$.

Proof. By assumption, there exist $u^{\varepsilon_{0}} \in L^{2}(0, T)$ such that, $\sup _{\theta \in \Theta}\left|\mathrm{x}_{\theta}^{1}-x_{\theta}\left(T ; \mathrm{x}_{\theta}^{0}, u^{\varepsilon_{0}}\right)\right| \leqslant \varepsilon_{0}$. It is also easy to see that $\left\|u_{K}^{\varepsilon_{0}}\right\|_{L^{2}(0, T)} \leqslant\left\|u^{\varepsilon_{0}}\right\|_{L^{2}(0, T)}$, hence, by application of Lemma 2.15, we obtain,

$$
\sup _{\theta \in \Theta}\left|\mathrm{x}_{\theta}^{1}-x_{\theta}\left(T ; \mathrm{x}_{\theta}^{0}, u_{K}^{\varepsilon_{0}}\right)\right| \leqslant \varepsilon_{0}+\frac{C_{0}+C_{1}\left\|u_{K}^{\varepsilon_{0}}\right\|_{L^{2}(0, T)}}{K-1} \leqslant \varepsilon_{0}+\frac{C_{0}+C_{1}\left\|u^{\varepsilon_{0}}\right\|_{L^{2}(0, T)}}{K-1} .
$$

It is then trivial to conclude the existence of $K\left(\varepsilon, \varepsilon_{0}\right) \in \mathbb{N}^{*}$ such that for every $K>K\left(\varepsilon, \varepsilon_{0}\right)$ we have, $\frac{C_{0}+C_{1}\left\|u^{\varepsilon_{0}}\right\|_{L^{2}(0, T)}}{K-1} \leqslant \varepsilon-\varepsilon_{0}$.

Remark 2.18. Let us also mention that, up to an extraction of a subsequence, the sequence of controls $\left(u_{K}^{\varepsilon_{0}}\right)_{K}$, given in Proposition 2.17. converges in $L^{2}$ to the control, $u^{\varepsilon_{0}}$, of minimal $L^{2}$-norm control such that $\sup _{\theta \in \Theta}\left|\mathrm{x}_{\theta}^{1}-x_{\theta}\left(T ; \mathrm{x}_{\theta}^{\sigma}, u^{\varepsilon_{0}}\right)\right|<\varepsilon_{0}$.

Indeed, for every $K$, we have $\left\|u_{K}^{\varepsilon_{0}}\right\|_{L^{2}(0, T)^{M}} \leqslant\left\|u^{\varepsilon_{0}}\right\|_{L^{2}(0, T)^{M}}$. Hence, there exist $u_{*}^{\varepsilon_{0}} \in L^{2}(0, T)^{M}$ such that up to an extraction of a subsequence, the sequence $\left(u_{K}^{\varepsilon_{0}}\right)_{K}$ is weakly convergent to $u_{\star}^{\varepsilon_{0}}$. In addition, we have $\left\|u_{*}^{\varepsilon_{0}}\right\|_{L^{2}(0, T)^{M}} \leqslant\left\|u^{\varepsilon_{0}}\right\|_{L^{2}(0, T)^{M}}$, and the weak convergence of $\left(u_{K}^{\varepsilon_{0}}\right)_{K}$ to $u_{*}^{\varepsilon_{0}}$ ensures that $\lim _{K \rightarrow \infty} x_{\theta}\left(T ; \mathrm{x}_{\theta}^{0}, u_{K}^{\varepsilon_{0}}\right)=\mathrm{x}_{\theta}\left(T ; \mathrm{x}_{\theta}^{0}, u_{\star}^{\varepsilon_{0}}\right)$. Furthermore, the two maps $\theta \mapsto x_{\theta}\left(T ; \mathrm{x}_{\theta}^{0}, u_{K}^{\varepsilon_{0}}\right)$ and $\theta \mapsto \mathrm{x}_{\theta}\left(T ; \mathrm{x}_{\theta}^{0}, u_{*}^{\varepsilon_{0}}\right)$ are both Lipschitz with the same Lipschitz constant, $C_{0}+C_{1}\left\|u^{\varepsilon_{0}}\right\|_{L^{2}(0, T)^{M}}$, 
with the notations of Lemma 2.15 . This, together with the compactness assumption on $\Theta$, ensures that $\sup _{\theta \in \Theta}\left|\mathrm{x}_{\theta}^{1}-x_{\theta}\left(T ; \mathrm{x}_{\theta}^{0}, u_{*}^{\varepsilon_{0}}\right)\right|=\lim _{K \rightarrow \infty} \sup _{\theta \in \Theta}\left|\mathrm{x}_{\theta}^{1}-x_{\theta}\left(T ; \mathrm{x}_{\theta}^{0}, u_{K}^{\varepsilon_{0}}\right)\right|$. This last fact, combined with the estimation $(2.26)$, leads to $\sup _{\theta \in \Theta}\left|\mathrm{x}_{\theta}^{1}-x_{\theta}\left(T ; \mathrm{x}_{\theta}^{0}, u_{*}^{\varepsilon_{0}}\right)\right| \leqslant \varepsilon_{0}$, and hence, the uniqueness of the minimal $L^{2}$-norm control implies $u_{*}^{\varepsilon_{0}}=u^{\varepsilon_{0}}$. Finally, since $\left\|u_{K}^{\varepsilon_{0}}\right\|_{L^{2}(0, T)^{M}} \leqslant\left\|u^{\varepsilon_{0}}\right\|_{L^{2}(0, T)^{M}}$, we also conclude that $\lim _{K \rightarrow \infty}\left\|u_{K}^{\varepsilon_{0}}\right\|_{L^{2}(0, T)^{M}}=\left\|u^{\varepsilon_{0}}\right\|_{L^{2}(0, T)^{M}}$, that is to say that $\left(u_{K}^{\varepsilon_{0}}\right)_{K}$ converges strongly in $L^{2}(0, T)^{M}$ to $u^{\varepsilon_{0}}$.

The Proposition 2.17 directly leads to the following algorithm to compute an appropriate control for ensemble controllability.

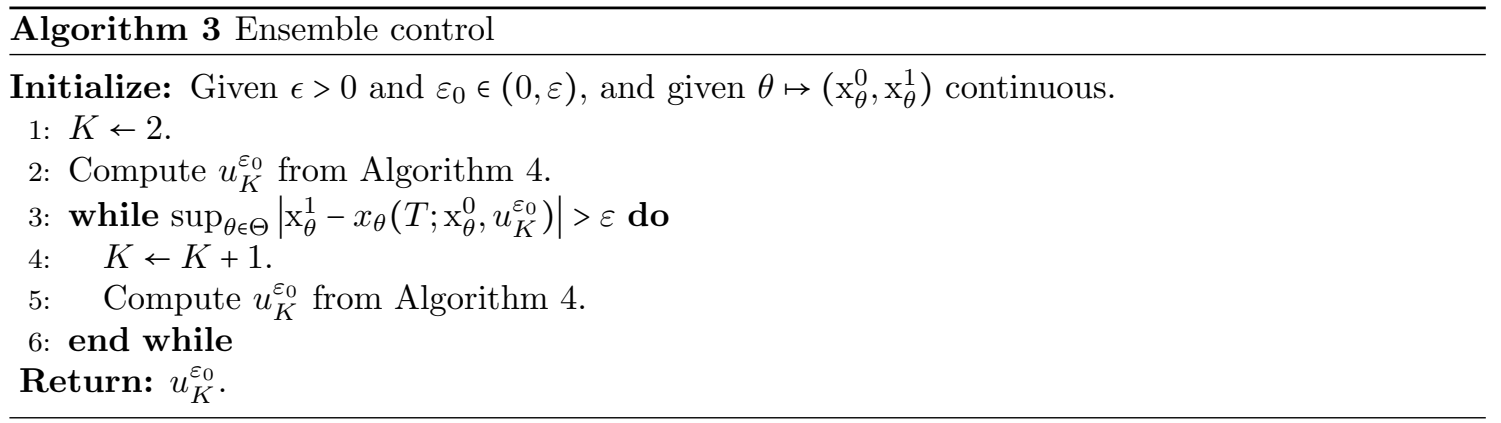

Remark 2.19. Obviously, in the $3^{\text {rd }}$ step of Algorithm 3 it is not possible to numerically compute $\sup _{\theta \in \Theta}\left|\mathrm{x}_{\theta}^{1}-x_{\theta}\left(T ; \mathrm{x}_{\theta}^{0}, u_{K}^{\varepsilon_{0}}\right)\right|$. Hence, one has to discretized $\Theta$ one again. This can be done, by picking some large number $L$ and some values $\widetilde{\theta}_{1}, \ldots, \widetilde{\theta}_{L} \in \Theta$, and substituting $\sup _{\theta \in \Theta}\left|\mathrm{x}_{\theta}^{1}-x_{\theta}\left(T ; \mathrm{x}_{\theta}^{0}, u_{K}^{\varepsilon_{0}}\right)\right|$ by $\sup _{l \in\{1, \ldots, L\}}\left|\mathrm{x}_{\widetilde{\theta}_{l}}^{1}-x_{\widetilde{\theta}_{l}}\left(T ; \mathrm{x}_{\widetilde{\theta}_{l}}^{0}, u_{K}^{\varepsilon_{0}}\right)\right|$. Note that the choice of the values of $\widetilde{\theta}_{l}$ can be random.

In Algorithm 3 , we use Algorithm 4, in order to compute the control of minimal $L^{2}$-norm such that 2.21) holds (with $\varepsilon$ replaced by $\varepsilon_{0}$ ).

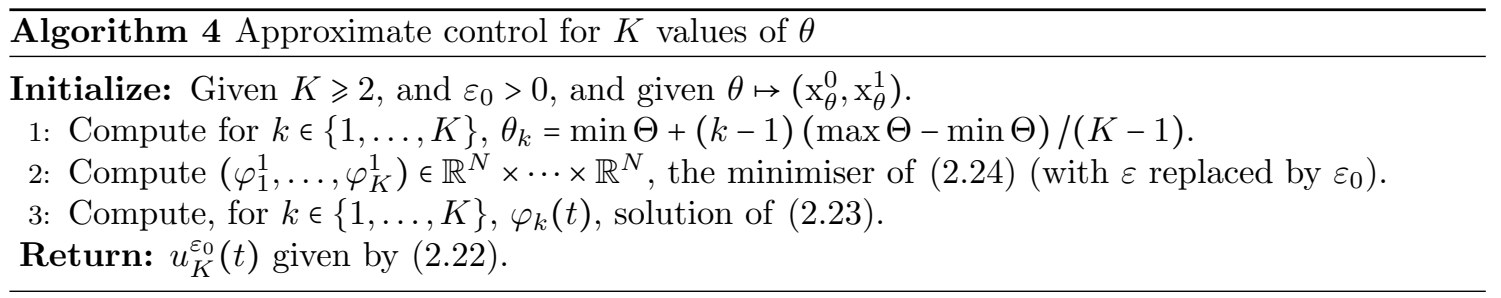

To conclude this subsection, we consider the following example.

Example 2.20. Let us consider $\Theta=[1,10]$, let us also assume that the dimension of the state is $N=2$ and the dimension of the control is $M=1$, we then consider the following matrices

$$
A_{\theta}=\left(\begin{array}{cc}
-1 & -\theta \\
\theta & -1
\end{array}\right) \quad \text { and } \quad B_{\theta}=\left(\begin{array}{l}
0 \\
1
\end{array}\right) \quad(\theta \in \Theta)
$$

With these matrices, we observe that the system (2.1) is ensemble controllable. Indeed, for every $\theta \in \Theta$, we have, $\sigma\left(A_{\theta}\right)=\{-1-i \theta,-1+i \theta\}$ and $\operatorname{rank}\left(B_{\theta}, A_{\theta} B_{\theta}\right)=\operatorname{rank}\left(\begin{array}{ll}0 & -\theta \\ 1 & -1\end{array}\right)=2$. It is then easy to see that items 13 of Proposition 2.14 are fulfilled and hence, the system (2.1), with the parameter set $\Theta$ and the matrices $A_{\theta}$ and $B_{\theta}$ defined above, is ensemble controllable. 
Let us now apply the result of Proposition 2.17. To this end, we consider the controllability time $T=2$, and the initial and final conditions given by $\mathrm{x}_{\theta}^{0}=(\theta \sin \theta)^{*}$ and $\mathrm{x}_{\theta}^{1}=0$. We then use Algorithm 4, with $\varepsilon_{0}=1 / 10$ and let $K \rightarrow \infty$. On Figure 2, we display the $L^{2}$-norm of the control $u_{K}$ and the maximum of $\theta \mapsto \mathrm{x}_{\theta}^{1}-x_{\theta}\left(T ; \mathrm{x}_{\theta}^{0}, u_{K}\right)$ for $K \in\{3, \ldots, 50\}$. To compute the maximum of $\left|\mathrm{x}_{\theta}^{1}-x_{\theta}\left(T ; \mathrm{x}_{\theta}^{0}, u_{K}\right)\right|$, we use Remark 2.19 with $L=5.10^{4}$ uniformly distributed values of $\theta$. We observe on this figure that $\left|\mathrm{x}_{\theta}^{1}-x_{\theta}\left(T ; \mathrm{x}_{\theta}^{0}, u_{K}\right)\right|$ decays to $\varepsilon_{0}$ as $K$ increase, we also observe that $\left\|u_{K}\right\|_{L^{2}(0, T)}$ is bouded. Note that, we cannot guarantee that $K \mapsto\left\|u_{K}\right\|_{L^{2}(0, T)}$ is increasing, and this is in general not the case, as wee see on Figure 2 However, we can ensure that $\left\|u_{2 K-1}\right\|_{L^{2}(0, T)} \geqslant\left\|u_{K}\right\|_{L^{2}(0, T)}$, this is due to the fact that the sequence of interpolation points for $2 K-1$ points contains the one for $K$ points.
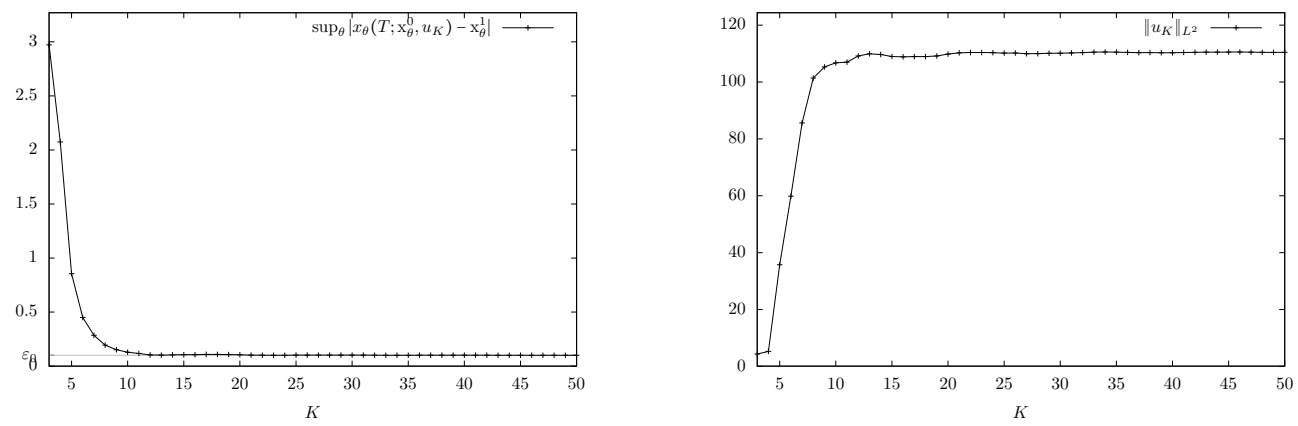

Figure 2: Display of $\left\|u_{K}^{\varepsilon_{0}}\right\|_{L^{2}(0, T)}$ and $\sup _{\theta \in \Theta}\left|x_{\theta}\left(T ; \mathrm{x}_{\theta}^{0}, u_{K}^{\varepsilon_{0}}\right)-\mathrm{x}_{\theta}^{1}\right|$, for $K \in\{3, \ldots, 50\}$, with $u_{K}$ given by Algorithm 4 for the ensemble control of the system considered in Example 2.20

\section{Parameter dependent controls}

Throughout this section we consider the system 1.1p accompanied by the control problem

$$
x_{\theta}(T)=\mathrm{x}_{\theta}^{1}, \quad(\theta \in \Theta),
$$

where the prescribed target state in general depends on the parameter, while the control time $T>0$, at which the system should reach the target, is $\theta$ independent. In addition, we suppose the system (1.1) is controllable for each value of the parameter $\theta \in \Theta$. In particular, it implies that for each given control time $T>0$ and the target $\mathrm{x}_{\theta}^{1}$ there exists a (parameter dependent) control such that the state of the system satisfies $x_{\theta}(T)=\mathrm{x}_{\theta}^{1}$. As explained in the introduction, such control (with minimal $L^{2}$-norm) can be explicitly constructed by solving the adjoint equation starting from optimal datum at time $T$ determined by the equation (3.3).

However, the construction of control for a large dimensional system is an expansive, time and computation consuming process. When dealing with parameter dependent problems, if this process is to be repeated many times, with every change of parameter value, we need to seek alternatives for solving the full problem over and over. For these reasons one tries to develop an approximate model of lower complexity providing the solution to problem of interest within a prescribed error. Such model we refer to as a reduced basis model.

An underlying assumption in application of reduced model techniques is that the solution manifold can be well approximated by a linear space of low dimension. In the setting of this chapter, this manifold consists of all controls (of minimal $L^{2}$-norm) of the system (1.1) obtained 
through variation of the parameter. Their implementation generally follows the offline-online paradigm. The purpose of the offline phase can be described as follows: given a Banach space $X$ and its compact subset $\mathcal{K}$, find a low-dimensional subspace $V \subset X$ such that $V$ approximates $\mathcal{K}$ fairly well. Once a reduced basis (of $V$ ) is determined, the approximation of an arbitrary element from $\mathcal{K}$ is calculated online as a linear combination of the reduced basis vectors. Normally, the cost of the offline routine is much higher and it requires large computational and memory capacities, but it can be considered as a pre-processing step that is performed only once.

The construction of the reduced basis mainly explores a proper orthogonal decomposition (POD) or a greedy sampling procedure, or their combination (POD-greedy) in case of timedependent problems [18, 22]. Here we also refer the reader to [43, Chapter 12] for a general introduction to reduced basis methods for parameter dependent control problems.

The former procedure consists of an explore-and-compress strategy, relying on a singular value decomposition of numerous snapshots (vectors selected from $\mathcal{K}$ ) and provide an optimal approximation space in the mean squared error sense. In the latter case the basis vectors coincide with the snapshots themselves, carefully selected through some optimality strategy, which significantly reduces the cost of the offline computation when compared to POD [22, 40. In addition, the greedy approach provides approximation rates comparable to the optimal ones, expressed through Kolmogorov widths.

By $u(\Theta)=\left\{u_{\theta}, \theta \in \Theta\right\}$ we denote the control manifold consisting of all optimal controls associated to problem (1.1) +3.1 as the parameter ranges over the whole set $\Theta$. As explained in the introduction, each such control can be uniquely determined by the relation

$$
u_{\theta}=B_{\theta}^{*} e^{(T-t) A_{\theta}^{*}} \varphi_{\theta}^{1},
$$

where $\varphi_{\theta}^{1}$ is the unique minimiser of the quadratic functional

$$
J_{\theta}\left(\varphi^{1}\right)=\frac{1}{2} \int_{0}^{T}\left|B_{\theta}^{*} \varphi(t)\right|^{2} d t-\left\langle\mathrm{x}_{\theta}^{1}, \varphi^{1}\right\rangle+\left\langle\mathrm{x}_{\theta}^{0}, \varphi(0)\right\rangle,
$$

and $\varphi$ is the solution to the adjoint system with datum at time $T$ given by $\varphi^{1}$.

Furthermore, this minimiser can be equivalently determined as the solution to the system

$$
\Lambda_{\theta} \varphi_{\theta}^{1}=\mathrm{x}_{\theta}^{1}-e^{T A_{\theta}} \mathrm{x}_{\theta}^{0},
$$

where $\Lambda_{\theta}$ is the controllability Gramian associated to $\left(A_{\theta}, B_{\theta}\right)$. The last system is well posed due to the assumed controllability property which implies that $\Lambda_{\theta}$ is a regular matrix. In particular, it defines the mapping $\theta \in \Theta \mapsto \varphi_{\theta}^{1} \in \mathbb{R}^{N}$, whose smoothness is transferred from the mappings of $A_{\theta}$ and $B_{\theta}$ at all levels. In the sequel by $\varphi^{1}(\Theta) \subset \mathbb{R}^{N}$ we denote the image of that mapping.

Having assumed these maps are Lipschitz continuous and the parameter $\theta \in \Theta$ varies on a compact set, both the set of all minimisers $\varphi^{1}(\Theta)$, as well as the set of corresponding optimal controls $u(\Theta)$ are compact sets. However, as the control manifold consists of time dependent functions, while the minimisers $\varphi_{\theta}^{1}$ are vectors from $\mathbb{R}^{N}$, we prefer to work in the latter framework and to construct an approximating subspace for the set $\varphi^{1}(\Theta)$. By using the one-to-one correspondence between the minimisers $\varphi_{\theta}^{1}$ and the associated controls $u_{\theta}$, this will in turn provide approximation of the control manifold as well. For these reasons we formulate our problem as follows.

1. Construct a reduced basis $V_{n}$ that approximates the manifold $\varphi^{1}(\Theta)$ within the given precision $\delta$.

2. For an arbitrary given parameter value $\theta \in \Theta$ construct an approximate control of the form $u_{\theta}^{\star}=B_{\theta}^{\star} e^{(T-t) A_{\theta}^{*}} \varphi_{\theta}^{1, \star}$, with $\varphi_{\theta}^{1, \star} \in V_{n}$ such that it steers the system 1.1 to the prescribed target $\mathrm{x}_{\theta}^{1}$ as close as possible. 
The first part of the problem corresponds to the offline phase, while the second one should enable efficient computation of an approximate control during the online procedure.

Of course, the problem is feasible due to the compactness of the parameter set $\Theta$ and the continuous-dependence assumption of dynamics with respect to $\theta$. However, the goal is to minimize the number of basis vectors $n$ and to obtain the most efficient approximation. The type of the approximation error (uniform, mean square, ... ) to be considered depends on the method selected for the construction of reduced basis, and will be discussed in the next subsection.

In the subsection devoted to the online phase we shall see how to construct approximation $\varphi_{\theta}^{1, \star}$ for a particular given parameter value, and discuss performance of the associated approximated controls.

\subsection{Selection of a reduced basis}

When dealing with a continuous range of parameter values, in order to do any kind of numerics, the first step requires discretization of the parameter set. From now on, by $\Theta_{h}$ we denote a finite set of cardinality $m=\left|\Theta_{h}\right|$ obtained by intersecting a regular lattice with $\Theta$ with the property

$$
\forall \theta \in \Theta \quad \operatorname{dist}\left(\theta, \Theta_{h}\right)<h .
$$

\subsubsection{Proper Orthogonal Decomposition (POD)}

Proper Orthogonal Decomposition is the reduced basis method that provides an optimal approximation space in the root-mean-square (RMS) error sense. Beside their numerous successful applications in different areas, here we just refer to those related to the control theory ([7, Chapter 1] and the references therein).

In general, for a finite set of vectors $\left\{y_{1}, \ldots, y_{m}\right\}$ in $\mathbb{R}^{N}$, POD results in the $n<m$ dimensional space that minimizes the quantity

$$
\sqrt{\frac{1}{m} \sum_{i} \operatorname{dist}\left(y_{i}, V_{n}\right)^{2}}
$$

over all $n$-dimensional subspaces $V_{n}$ of the $\operatorname{Span}\left\{y_{1}, \ldots, y_{m}\right\}$.

To construct the POD space one introduces the correlation matrix $C=\frac{1}{m} Y^{*} Y \in \mathbb{R}^{N \times N}$, where $Y$ is the matrix consisting of column vectors $y_{i}, i \in\{1, \ldots, m\}$. Then the (orthogonal) POD basis $V_{n}$ consists of eigenvectors of $C$ corresponding to its largest $n$ eigenvalues $\lambda_{i}$ (sorted in a decreasing order), and it satisfies the following estimate

$$
\frac{1}{m} \sum_{i} \operatorname{dist}\left(y_{i}, V_{n}\right)^{2}=\sum_{i=n+1}^{N} \lambda_{i}
$$

where we sum the remaining eigenvalues of the correlation matrix.

Thus, the construction of the POD basis corresponds to the singular value decomposition (SVD) of the matrix $\frac{1}{\sqrt{m}} Y$.

In the context of parameter dependent control problems, we formulate the corresponding algorithm as follows.

The estimate (3.4) implies directly that the subspace $V_{n}$ constructed by Algorithm 5 approximates the set $\varphi^{1}\left(\Theta_{h}\right)=\left\{\varphi_{\theta}^{1} \mid \theta \in \Theta_{h}\right\}$ with the RMS error less than $\delta / \sqrt{2}$. In addition, the above algorithm provides the mean square approximation over the whole range of parameter set $\Theta$, i.e., one obtains an estimate on the expected value of $\operatorname{dist}\left(\varphi_{\theta}^{1}, V_{n}\right)^{2}$, where we assume that $\theta$ obeys some probability distribution law. 


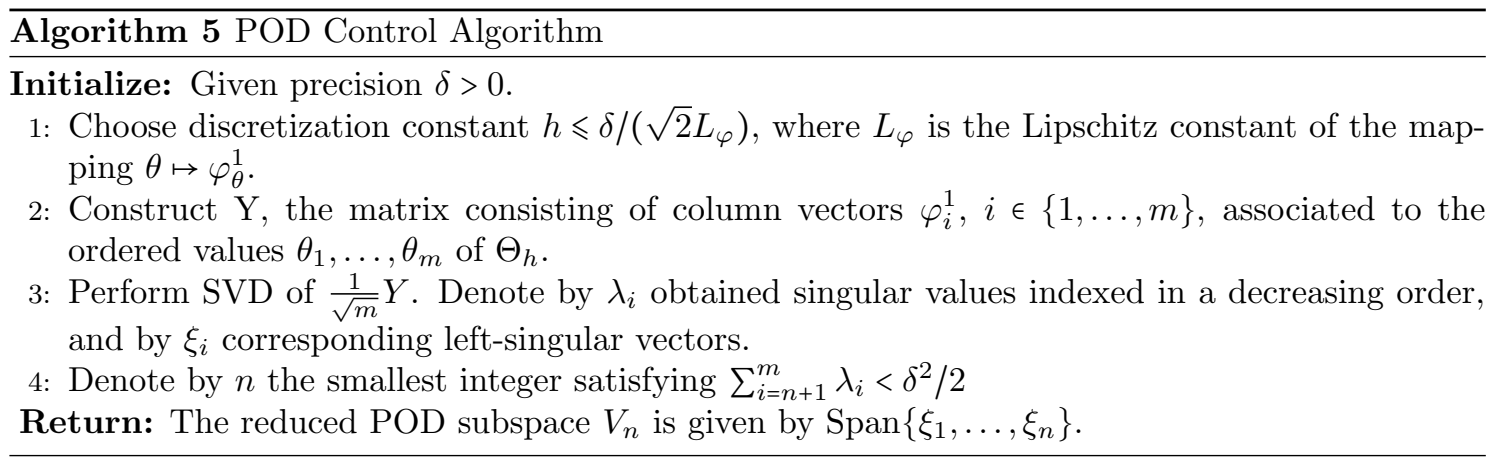

For simplicity let us assume that $\theta$ is uniformly distributed over the set $\Theta$. Let us denote by $\left(\Theta_{i}\right)_{i \in\{1, \ldots, m\}}$ a disjoint union of $\Theta$ consisting of equal measure sets centered around $\theta_{i} \in \Theta_{h}$. More precisely we suppose $\Theta_{i} \subseteq B\left(\theta_{i}, h\right)$ (where $h$ is the discretization constant from Algorithm 5) and $\left|\Theta_{i}\right|=1 / m$ for every index $i$. Then

$$
\begin{aligned}
E\left(\operatorname{dist}\left(\varphi_{\theta}^{1}, V_{n}\right)^{2}\right) & =\frac{1}{|\Theta|} \int_{\Theta} \operatorname{dist}\left(\varphi_{\theta}^{1}, V_{n}\right)^{2} \mathrm{~d} \theta \\
& \leqslant \frac{1}{|\Theta|} \sum_{i} \int_{\Theta_{i}}\left|\varphi_{\theta}^{1}-\varphi_{i}^{1}\right|^{2} \mathrm{~d} \theta+\frac{1}{m} \sum_{i} \operatorname{dist}\left(\varphi_{i}^{1}, V_{n}\right)^{2} .
\end{aligned}
$$

By exploring the Lipschitz continuity of the mapping $\theta \mapsto \varphi_{\theta}^{1}$ and the choice of the discretization constant $h$ it follows

$$
\left|\varphi_{\theta}^{1}-\varphi_{\theta_{i}}^{1}\right|^{2} \leqslant L_{\varphi}\left|\theta-\theta_{i}\right|^{2} \leqslant \delta^{2} / 2
$$

Finally, combining the last estimate with the RMS error bound of the set $\varphi^{1}\left(\Theta_{h}\right)$ we obtain

$$
\sqrt{E\left(\operatorname{dist}\left(\varphi_{\theta}^{1}, V_{n}\right)^{2}\right)} \leqslant \delta
$$

Remark 3.1. It should be noted that the Lipschitz constant $L_{\varphi}$ for a general system might not be available in practice. However, as there exists an explicit expression for $\varphi_{\theta}$ (cf. (3.3)), $L_{\varphi}$ can be bounded from above in terms of the Lipschitz constants of the mappings $\theta \rightarrow\left(A_{\theta}, B_{\theta}, \mathrm{x}_{\theta}^{0}, \mathrm{x}_{\theta}^{1}\right)$. Thus, for specific examples, where the dependence of the system dynamics and data on the parameter is given explicitly, one can calculate the latter constants, and use the upper bound for $L_{\varphi}$ in order to estimate an optimal value of the discretization constant $h$.

In general, when we lack an estimate on $L_{\varphi}$, Algorithm 5 still provides the approximation over the discretized parameter set $\Theta_{h}$, while the discretization constant $h$ has to be chosen arbitrarily small.

\subsubsection{Greedy algorithm}

One of the most popular methods for constructing reduced basis relies on the so called greedy algorithms. These were successfully introduced and analysed in works of A. Patera and his collaborators two decades ago (cf. 49]) with the aim of solving parametric PDEs. Later they were applied to optimal control problems [20, 23, 24, while the application to controllability of linear systems was introduced in [31.

The method provides a linear subspace of a relatively small dimension that approximates a compact subset $\mathcal{K}$ in a Banach space $X$ with a given precision $\delta$. A general greedy algorithm is given in Algorithm 6 below. 


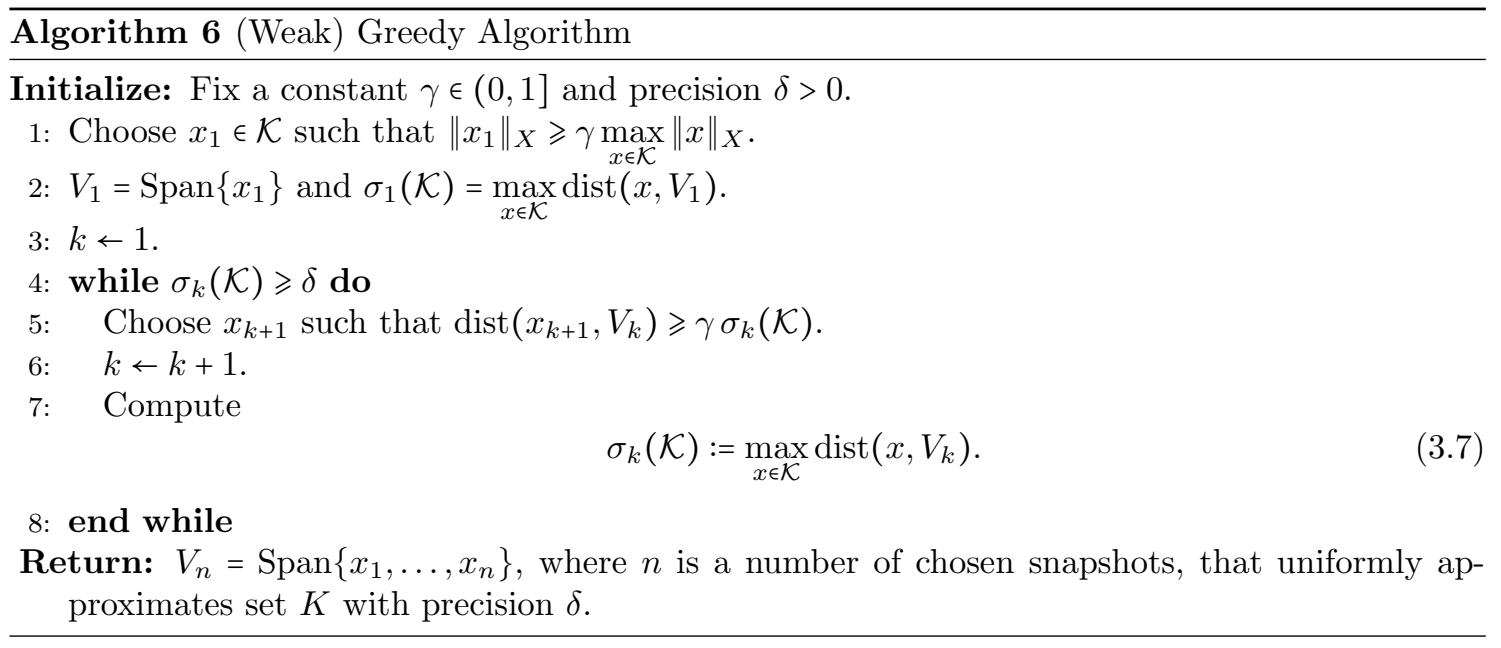

The choice of the constant $\gamma$ equal to one corresponds to the pure greedy algorithm. Reduction of its value simplify implementation of the algorithm as the optimization problem (3.7) does not have to be solved fully but only up to some fixed percentage extent. On the other side, as we shall see below, the relaxation of the pure greedy algorithm to a weak greedy (with $\gamma \in(0,1)$ ) does not significantly reduce the efficiency of the algorithm.

One of great advantages of greedy algorithms is their approximation efficiency, which is comparable with the optimal ones expressed through Kolmogorov widths. The latter is defined as

$$
d_{n}(\mathcal{K}):=\inf _{\operatorname{dim} Y=n} \sup _{x \in \mathcal{K}} \inf _{y \in Y}\|x-y\|_{X},
$$

and they measure how well $\mathcal{K}$ can be approximated by a subspace in $X$ of a fixed dimension $n$.

The following theorem (taken from [13, Corollary 3.3]) gives a comparison of $\sigma_{n}(\mathcal{K})$ with $d_{n}(\mathcal{K})$.

Theorem 3.2. For the weak greedy algorithm with constant $\gamma \in(0,1]$ in a Hilbert space $X$, we have the following: If the compact set $\mathcal{K}$ is such that, for some $\alpha>0$ and $C_{0}>0$

$$
d_{n}(\mathcal{K}) \leqslant C_{0} n^{-\alpha} \quad(n \in \mathbb{N}),
$$

then

where $C_{1}:=\gamma^{-2} 2^{5 \alpha+1} C_{0}$.

$$
\sigma_{n}(\mathcal{K}) \leqslant C_{1} n^{-\alpha} \quad(n \in \mathbb{N}),
$$

This theorem implies that in the case of a polynomial decay the (weak) greedy algorithms perform with the same rate as Kolmogorov widths. A similar estimate holds for subexponential decays as well (cf. [13]). Note that the weak greedy constant $\gamma$ does not influence decay rates, as it enters the above estimate only through the multiplicative constant $C_{1}$.

However, in practical implementations, the compact set $\mathcal{K}$ to be approximated is often unknown (e.g. it consists of solutions to parameter dependent problems, as in our case) which poses a significant challenge when dealing with the maximisation problem (3.7). Actually, it requires to evaluate the distance of a vector from the constructed reduced space without knowing that vector! Of course, computing all the elements of the set $\mathcal{K}$ is not a remedy, as it would require to solve a problem for each parameter value what is exactly we want to avoid. In order to solve this issue, instead of calculating the exact distance appearing in (3.7), one use some surrogate distance which is easier to compute. 
The next theorem demonstrates that such replacement still provides a greedy approximation of the set $\mathcal{K}$ and it only affects value of the weak greedy constant $\gamma$.

Theorem 3.3. Let $f: X \times X \mapsto \mathbb{R}$ be a function satisfying

$$
c\|x-y\|_{X} \leqslant f(x, y) \leqslant C\|x-y\|_{X} \quad(x, y \in \mathcal{K}),
$$

for some constants $C, c>0$. Perform the Algorithm 6 with the distance $\operatorname{dist}\left(x, V_{k}\right)$ replaced by $\inf _{y \in V_{k}} f(x, y)$. Then the such modified algorithm provides a weak greedy search of the set $\mathcal{K}$ with the weak greedy constant $\gamma^{\prime}=\gamma c / C$.

Proof. In order to demonstrate the theorem it is enough to show that the vectors selected by the modified algorithm in each step satisfy

$$
\operatorname{dist}\left(x_{k+1}, V_{k}\right) \geqslant \gamma^{\prime} \max _{x \in \mathcal{K}} \operatorname{dist}\left(x, V_{k}\right) .
$$

To this end note that for any $x \in \mathcal{K}$ we have

$$
\begin{aligned}
c \operatorname{dist}\left(x, V_{k}\right) & \leqslant \inf _{y \in V_{k}} f(x, y) \\
& \leqslant \frac{1}{\gamma} \inf _{y \in V_{k}} f\left(x_{k+1}, y\right) \leqslant \frac{C}{\gamma} \operatorname{dist}\left(x_{k+1}, V_{k}\right),
\end{aligned}
$$

where the middle inequality results from the selection criteria of the modified algorithm for the next reduced basis vector $x_{k+1}$, while the remaining two from the equivalence relation (3.8).

Therefore, for practical implementations of (weak) greedy algorithms it is essential to detect a surrogate distance which is easy to compute even without knowing the vectors itself.

In the context of control problems for parameter dependent systems, the set to be approximated is the manifold $\varphi^{1}(\Theta)$ that consists of all minimisers of a functional $J_{\theta}$ given by 3.2 as the parameter ranges over the compact set $\Theta$. Of course, we do not have these minimisers at our disposal, thus we need to find a surrogate that will enable implementation of the greedy algorithm.

Let us suppose that $\varphi_{1}^{1}$ is a reduced basis vector selected through the weak greedy search of $\varphi^{1}(\Theta)$. In order to estimate distance of other vectors from the space spanned by $\varphi_{1}^{1}$, note that for any $\theta \in \Theta$ we have

$$
\Lambda_{-}\left\|\varphi_{\theta}^{1}-\varphi_{1}^{1}\right\| \leqslant\left\|\Lambda_{\theta} \varphi_{\theta}^{1}-\Lambda_{\theta} \varphi_{1}^{1}\right\| \leqslant \Lambda_{+}\left\|\varphi_{1}^{1}-\varphi_{1}^{1}\right\|
$$

where the uniform coercive and boundedness constants on the Gramian operators (i.e. $\Lambda_{-} I \leqslant \Lambda_{\theta} \leqslant$ $\Lambda_{+} I$ for every $\theta \in \Theta$ ) follow from the compactness of the set $\Theta$ and the regularity of mappings $\theta \mapsto A_{\theta}, B_{\theta}$.

Although this note provides $\left\|\Lambda_{\theta} \varphi_{\theta}^{1}-\Lambda_{\theta} \varphi_{1}^{1}\right\|$ as a surrogate distance of $\varphi_{\theta}^{1}$ from $\varphi_{1}^{1}$, it will turn useful only if we can calculate it (much) easier than the exact one. However, by using characterisation of the optimal adjoint datum (3.3) we know that $\Lambda_{\theta} \varphi_{1}^{1}$ can be expressed in terms of the corresponding initial and target data, which are prescribed in advance, i.e., we have them at our disposal. As for the other term entering the expression of the surrogate distance, it represents the final state of the system corresponding to the parameter value $\theta$ run by a control of the form $u=B_{\theta}^{*} \varphi$, where $\varphi$ is the solution of the adjoint system with datum at time $T$ equal to $\varphi_{1}^{1}$. In other worlds, $\Lambda_{\theta} \varphi_{1}^{1}$ is a residual obtained by replacing the optimal adjoint datum $\varphi_{\theta}^{1}$ by a reduced basis vector (Figure 3). Its calculation thus requires solving consecutively the adjoint plus primal system, which of course is much cheaper than calculating $\varphi_{\theta}^{1}$.

Based on the above discussion we propose the greedy control algorithm (Algorithm 7) as follows, see 31. 


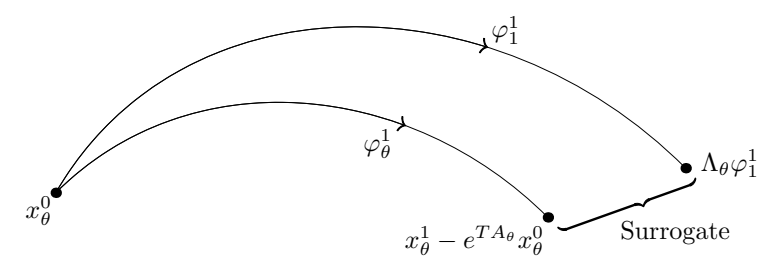

Figure 3: The surrogate of $\operatorname{dist}\left(\varphi_{\theta}^{1}, \varphi_{1}^{1}\right)$.

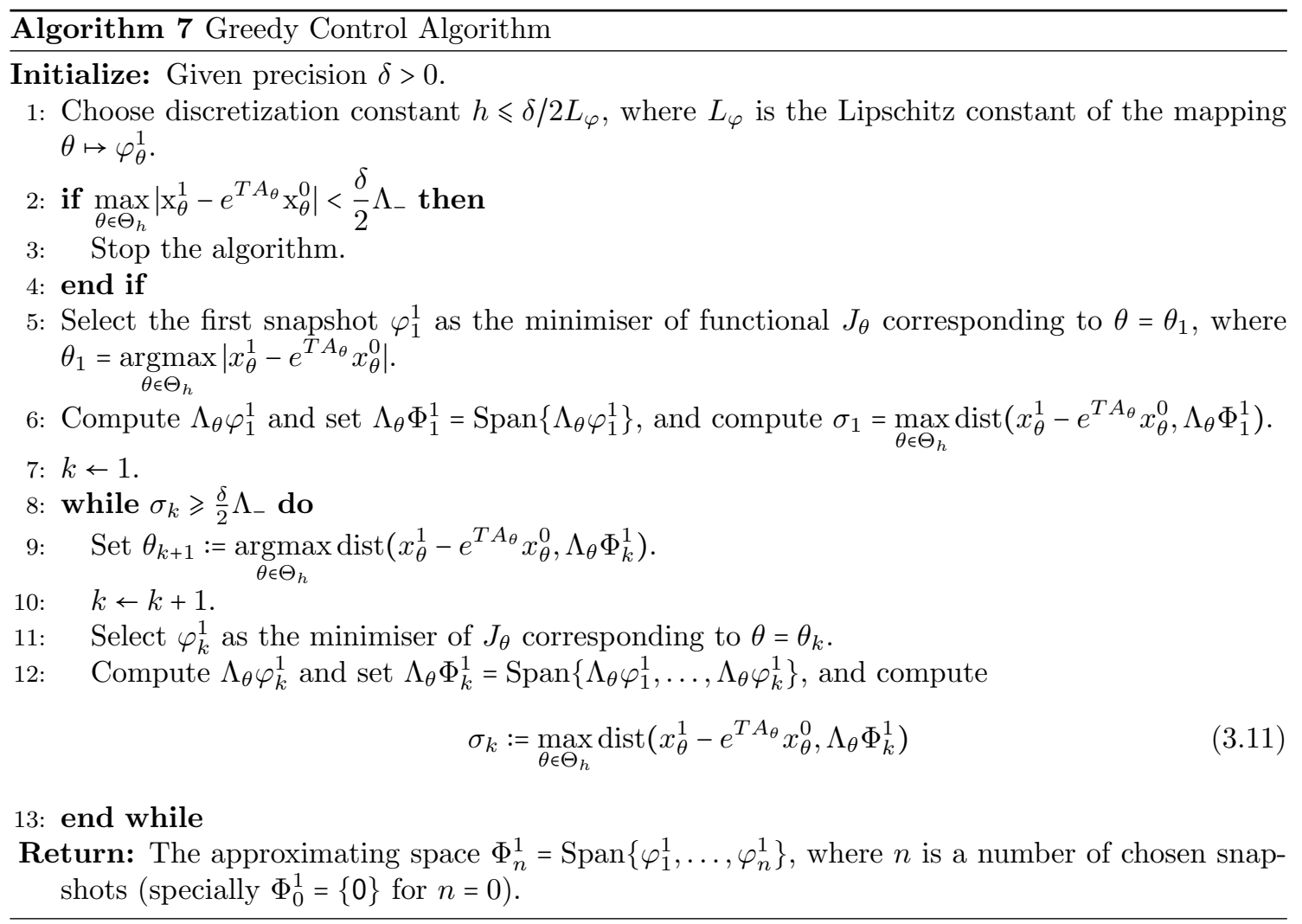

Obviously, the set $\left\{\varphi_{1}^{1}, \ldots, \varphi_{n}^{1}\right\}$ is linearly independent due to the selection criteria (3.11). Furthermore, as it consisis of vectors in $\mathbb{R}^{N}$, the algorithm stops after, at most, $n \leqslant N$ iterations, and it fulfils the requirements of the weak greedy theory. More precisely Theorem 3.4 holds.

Theorem 3.4 (Theorem 4.1 of 31). Algorithm 7 provides a week greedy approximation of the manifold $\varphi^{1}(\Theta)$ with the constant $\gamma=\Lambda_{-} /\left(2 \Lambda_{+}\right)$and the uniform approximation error less than $\delta$.

Comparing the last algorithm with the POD method, we note that the first requires solving the control problem only for $n$ chosen parameter values, for which the corresponding minimisers of a functional $J_{\theta}$ given by $(3.2$ constitute the reduced basis. On the other hand, the latter method requires the optimization problem for $(3.2$ to be solved for every parameter value from the training set $\Theta_{h}$, and eventually extracts a small number of most important directions out of collected solutions. Although the greedy selection, in addition to construction of the reduced basis vectors, requires computation of residual in each iteration for all parameter values from $\Theta_{h}$, its total cost is still cheaper than the cost of calculating $\varphi_{\theta}^{1}$ for all values of $\Theta_{h}$ (a precise estimate 
on its computational cost can be found in 31]), which makes the greedy control strategy more efficient than the POD one.

Of course, existence of an affordable surrogate (given by (3.10) plays an essential role in the above analysis. For general problems, that might lack an appropriate surrogate, the choice of an optimal method is not straightforward, and depends on particular characteristics of each problem of interest.

Remark 3.5. Theorem 3.4 remains valid if instead of Lipschitz continuity we merely assume continuous dependence with respect to the parameter. Namely, as $\Theta$ is a compact set, the assumption directly implies uniform continuity, which suffices for the proof of Theorem 3.4 . The only difference in that case is that the discretization constant $h$ can not be given explicitly in terms of precision $\delta$, unlike it was given in Algorithm 7. This is also important in the case when the Lipschitz constant $L_{\varphi}$ might not be available (cf. Remark 3.1).

\subsection{The online procedure}

In this subsection, given any parameter value $\theta \in \Theta$, we aim to build an approximation $\varphi_{\theta}^{1, \star}$ of an optimal adjoint datum by selecting an appropriate linear combination of reduced basis vectors. The selection criteria is prescribed by the Problem formulation, where the ultimate goal is to reach the prescribed target $x_{\theta}^{1}$ as close as possible. Hereby we assume that we have at our disposal a reduced basis space $V_{n}=\left[\varphi_{1}^{1}, \ldots, \varphi_{n}^{1}\right]$ constructed in the offline phase by some of the methods explained above.

The state of the system at final time $T$ driven by the approximate control determined by $\varphi_{\theta}^{1, \star}$ is given by

$$
x_{\theta}^{\star}(T)=\Lambda_{\theta} \varphi_{\theta}^{1, \star}+e^{T A_{\theta}} \mathbf{x}_{\theta}^{0},
$$

By exploring relation 3.3 we see that its distance from the prescribed target equals

$$
\left|\mathrm{x}_{\theta}^{1}-x_{\theta}^{\star}(T)\right|=\left|\Lambda_{\theta}\left(\varphi_{\theta}^{1}-\varphi_{\theta}^{1, \star}\right)\right|,
$$

and it reaches the minimal value if $\varphi_{\theta}^{1, \star}$ is selected as the solution to the problem

$$
\varphi_{\theta}^{1, \star}=\underset{\varphi^{1} \in V_{n}}{\operatorname{argmin}}\left\|\Lambda_{\theta}\left(\varphi_{\theta}^{1}-\varphi^{1}\right)\right\|=\underset{\varphi^{1} \in V_{n}}{\operatorname{argmin}}\left\|\left(\mathrm{x}_{\theta}^{1}-e^{T A_{\theta}} \mathrm{x}_{\theta}^{0}\right)-\Lambda_{\theta} \varphi^{1}\right\|
$$

This choice of $\varphi_{\theta}^{1, \star}$ corresponds to the minimization of the functional $J_{\theta}$, determined by 3.2 , over the space $V_{n}$.

The minimum in 3.12 is obtained if $\Lambda_{\theta} \varphi_{\theta}^{1, \star}$ equals the projection of $\mathrm{x}_{\theta}^{1}-e^{T A_{\theta}} \mathrm{x}_{\theta}^{0}$ to the space $\operatorname{Span}\left\{\Lambda_{\theta} \varphi_{1}^{1}, \ldots, \Lambda_{\theta} \varphi_{n}^{1}\right\}$. As $\Lambda_{\theta}$ is a linear operator, the coefficients of that projection in the latter basis will also provide the optimal choice of $\varphi_{\theta}^{1, \star}$.

For these reasons we formulate the online algorithm as follows (see [31]).

Remark 3.6. Note that for a parameter value $\theta$ that belongs to the discretization set $\Theta_{h}$ used in the construction of the reduced basis space $V_{n}$ some steps of the last algorithm can be skipped as the corresponding terms have already been calculated within the offline part of the algorithm. In particular, steps 1-3 in the case of a greedy selection of $V_{n}$, while for $\theta \in \Theta_{h}$ the whole algorithm turns superfluous if the POD method was used in the offline phase. As explained above, this is due to the specific characteristic of the latter approach that first explores the solution over a large number parameter, and subsequently compress relevant information out of it in order to construct the reduced basis. 


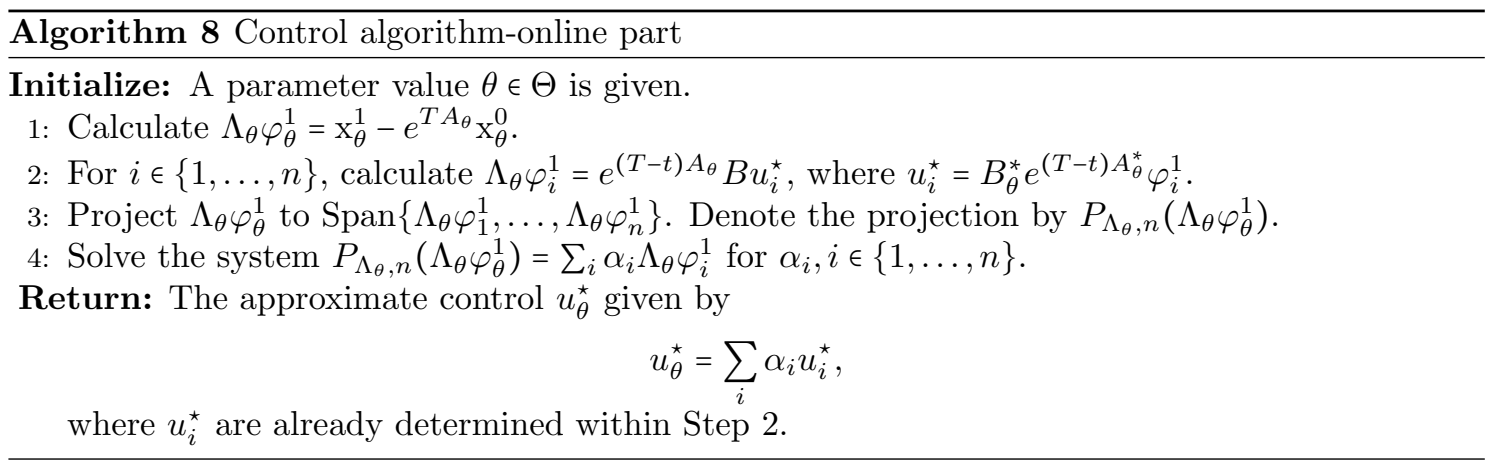

Algorithm 8 differs from the standard online procedure used in reduced basis methods (cf. [22]). The latter choose the approximate solution to the problem as its Galerkin projection to the selected reduced basis. In particular, in our setting this would determine an approximate adjoint datum as Galerkin projection to $\operatorname{Span}\left\{\varphi_{1}^{1}, \ldots, \varphi_{n}^{1}\right\}$ considered as solution to $\Lambda_{\theta} \varphi_{\theta}^{1}=\mathrm{x}_{\theta}^{1}-e^{T A_{\theta}} \mathrm{x}_{\theta}^{0}$. More precisely it would result in a vector $\varphi_{\theta}^{1, \mathrm{GAL}}=\sum \alpha_{i}^{\mathrm{GAL}} \varphi_{i}^{1}$, where the coefficients $\alpha_{i}^{\mathrm{GAL}}$ are chosen as solution to the system

$$
\sum_{i}\left\langle\Lambda_{\theta} \varphi_{i}^{1}, \varphi_{j}^{1}\right\rangle \alpha_{i}^{\mathrm{GAL}}=\left\langle\mathrm{x}_{\theta}^{1}-e^{T A_{\theta}} \mathrm{x}_{\theta}^{0}, \varphi_{j}^{1}\right\rangle
$$

On the other hand, the coefficients $\alpha_{i}$ obtained by Algorithm 8 are determined by orthogonal projection of $\mathrm{x}_{\theta}^{1}-e^{T A_{\theta}} \mathrm{x}_{\theta}^{0}$ to the $\operatorname{Span}\left\{\Lambda_{\theta} \varphi_{1}^{1}, \ldots, \Lambda_{\theta} \varphi_{n}^{1}\right\}$, and they satisfy the system

$$
\sum_{i}\left\langle\Lambda_{\theta} \varphi_{i}^{1}, \Lambda_{\theta} \varphi_{j}^{1}\right\rangle \alpha_{i}=\left\langle\mathrm{x}_{\theta}^{1}-e^{T A_{\theta}} \mathrm{x}_{\theta}^{0}, \Lambda_{\theta} \varphi_{j}^{1}\right\rangle .
$$

The reason for specific design of the online control algorithm lies in the particular goal of our problem - that is to steer the system to the prescribed target as close as possible, and the proposed algorithm ensures the best control performance.

Finally, the precise estimates on the deviation of the system from the target follows from estimates on the reduced basis approximation developed through the online phase. Indeed, as $\varphi_{\theta}^{1, *}$ is the solution to the minimization problem 3.12 it follows

$$
\begin{aligned}
\left|\mathrm{x}_{\theta}^{1}-x_{\theta}^{\star}(T)\right| & =\left|\Lambda_{\theta}\left(\varphi_{\theta}^{1}-\varphi_{\theta}^{1, \star}\right)\right| \\
& \leqslant\left|\Lambda_{\theta}\left(\varphi_{\theta}^{1}-P_{n} \varphi_{\theta}^{1}\right)\right| \leqslant \Lambda_{+}\left|\left(\varphi_{\theta}^{1}-P_{n} \varphi_{\theta}^{1}\right)\right|,
\end{aligned}
$$

where $P_{n}$ denotes the projection to the reduced basis space $V_{n}$, while $\Lambda_{+}$is a positive constant satisfying $\Lambda_{\theta} \leqslant \Lambda_{+} I$ for every $\theta \in \Theta$. Note that the finitude of $\Lambda_{+}$follows from the assumed Lipschitz dependence on the parameter and compactness of the set $\Theta$.

For these reasons, the estimates obtained in the offline procedure on the approximation of the manifold $\varphi^{1}(\Theta)$ by the reduced basis transfer to the online phase. In particular, taking into account (3.6), for the POD selection we obtain the RMS bound

$$
\sqrt{E\left(\left|\mathrm{x}_{\theta}^{1}-x_{\theta}^{\star}(T)\right|^{2}\right)} \leqslant \Lambda_{+} \delta .
$$

Similarly, the uniform estimate obtained by the greedy procedure provides

$$
\left|\mathrm{x}_{\theta}^{1}-x_{\theta}^{\star}(T)\right| \leqslant \Lambda_{+} \delta \quad(\theta \in \Theta) .
$$

The last two estimates allows one to carefully design the whole process (including both the offline and online phase) in order to ensure a desired control performance. In particular, if we want to 
steer the system to the prescribed target within the precision $\varepsilon$, than the reduced basis error should satisfy $\delta \leqslant \varepsilon / \Lambda_{+}$.

We finish this subsection by a numerical example.

Example 3.7. We consider the control system 1.1 with the governing matrix of the form $A_{\theta}=$ $\theta(N+1)^{2} \widetilde{A}$, where

$$
\widetilde{A}=\left(\begin{array}{cccccc}
-2 & 1 & 0 & \cdots & 0 & 0 \\
1 & -2 & 1 & \cdots & 0 & 0 \\
0 & 1 & -2 & \cdots & 0 & 0 \\
\vdots & \vdots & \vdots & \ddots & \vdots & \vdots \\
0 & 0 & 0 & \cdots & -2 & 1 \\
0 & 0 & 0 & \cdots & 1 & -2
\end{array}\right) \in \mathbb{R}^{N \times N}
$$

The control operator is assumed to be of the parameter-independent form

$$
B=\left(0, \ldots, 0,(N+1)^{2}\right)^{*} \in \mathbb{R}^{N} .
$$

Such system corresponds to the space-discretisation of the heat equation problem with $N$ internal grid points and the control on the right boundary:

$$
\left\{\begin{aligned}
\partial_{t} v-\theta \partial_{x x} v & =0, & & (t, x) \in(0, T) \times(0,1), \\
v(t, 0) & =0, & & t \in(0, T) \\
v(t, 1) & =u_{\theta}(t), & & t \in(0, T) \\
v(0, x) & =v_{0}(x), & & x \in(0,1) .
\end{aligned}\right.
$$

The parameter $\theta$ represents the diffusion coefficient and is supposed to range within the set $\Theta=$ $[1,10]$. We aim to control the system from the initial state $v_{0}(x)=\sin (\pi x)$ to zero in time $T=1 / 10$.

The offline greedy algorithm has been applied for the system of dimension $N=100$ with the stopping constant $\Lambda_{-} \delta / 2=10^{-4}$, and the uniform discretisation of $\Theta$ in $k=100$ values. The algorithm stops after only three iterations, choosing parameter values (out of 100 eligibles) in the following order:

$$
1.00,1.18,1.45 \text {. }
$$

The corresponding three minimisers $\varphi_{i}^{1}$ constitute the reduced basis $V_{3}$, and the approximate controls corresponding to all other parameter values are constructed as their suitable linear combination during the online phase. A rather small number of snapshots required to obtain approximation performance within the given accuracy is partially due to the strong dissipation effect of the heat equation, providing an exponential solution decay even in the absence of any control.

Here we present the results of the online algorithm applied for a specific parameter value $\theta=\pi$.

The corresponding approximate control is ploted on Figure 4a. It exhibits rather strong oscillations when approaching the final time which is an intrinsic feature for the heat equation. The elapsed time of the online procedure was 3.58 seconds only, compared to 286 seconds required to construct the optimal exact control. This huge difference is due to a rather small dimension of the reduced basis, and it reconfirms computational efficiency of the greedy approach when compared to the standard one.

Evolution of the solution, presented by a 3-D plot is given by Figure $4 \mathrm{~b}$. The system is driven to the zero state, as required, within the error $|x(T)|=1 \cdot 10^{-6}$.

\subsection{Other approaches}

Throughout this section we have dealt with a problem of constructing a reduced basis of the set $\varphi^{1}(\Theta)$ of all minimisers of the functional $J_{\theta}$, given by 3.2 , as $\theta$ ranges over the compact 


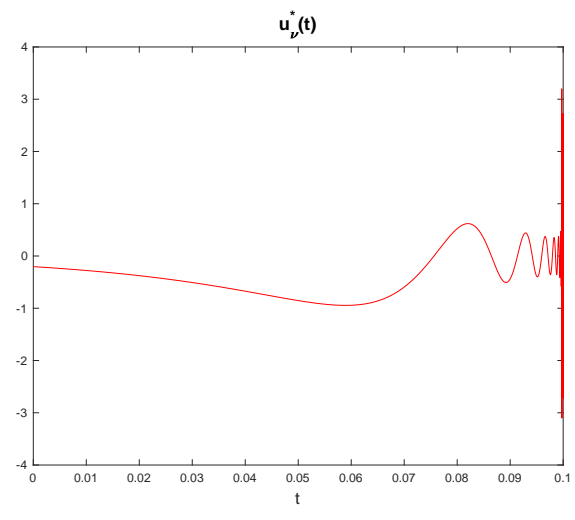

(a)

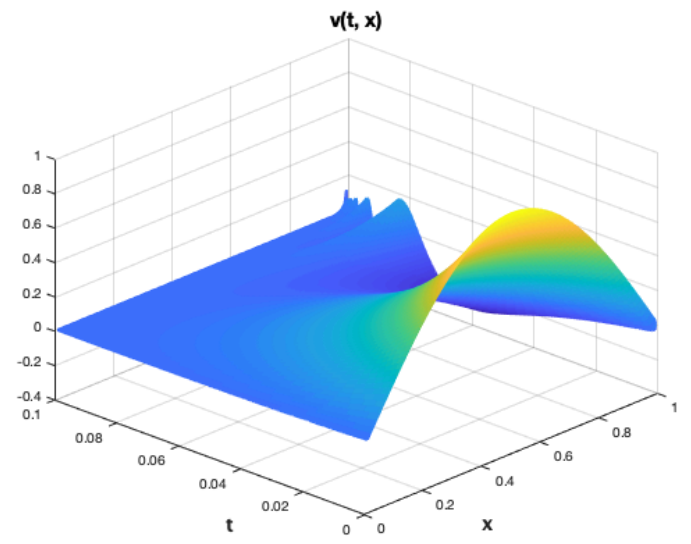

(b)

Figure 4: Evolution of the approximate control (a) and of the solution to the semi-discretised problem (3.15) governed by the approximate control $u_{\theta}^{\star}$ (b) for $\theta=\pi$.

set $\Theta$. By exploring the one-to-one correspondence between the minimisers $\varphi_{\theta}^{1}$ and the associated optimal controls $u_{\theta}$ such approach enables rapid approximation of the latter during the online stage.

Of course, there are other methods at disposal by which one can construct (approximatively) a control function steering the system of interest under particular circumstances, determined by the exact realisation of the parameter, to a prescribed target.

Construction of reduced basis for $u(\Theta)$. As an example, instead of dealing with the set $\varphi^{1}(\Theta)$, one can apply the reduced basis approach to the control manifold directly. A suggested method in that case would rely on a POD-Greedy strategy. This one is explored in case of time-dependent problems and it successfully combines two, above defined procedures (e.g. [18). In particular, each greedy step is accompanied by a corresponding POD process. The first selects a snapshot, which is a solution to time dependent problem for a specific parameter value, while the latter selects a single vector that approximates in the optimal manner the values of the snapshot evaluated at different time instants. It is important to mention that the method preserves convergences rates of greedy algorithms. More precisely, it maintains algebraic convergence rates of the Kolmogorov $n$-widths with the same exponent, and preserves their exponential decay with a slightly lower exponent (cf. [18, Section 4.2]).

The POD-Greedy approach has been applied to evolutional equations and parabolic control problems (e.g. [22, 23]), but, as far as we know, it has not yet been developed in the context of controllability.

Construction of reduced basis for $\Lambda(\Theta)$. The analysis and methods discussed so far apply to control problems where the initial and target data are prescribed a priori, either depending on the parameters or not. In particular, the above presented algorithms provide a reduced basis for a particular data selection, and each change of it requires running the algorithm from scratch again.

From the point of view of applications it would be therefor interesting to develop efficient methods for control of parameter dependent systems that are robust with respect to these data. This requires construction of a reduced basis for the approximation of the Gramians $\Lambda_{\theta}$, within the space of bounded linear operators.

A development of appropriate greedy procedure in that case, as explained above, requires 
identification of a feasible and easy executable surrogate. The key point in that direction is that Gramian operators satisfy a linear matrix relation: a differential Lyapunov equation in a case of a finite time Gramian, or an algebraic Lyapunov one for an infinite time horizon. In particular, the infinite time Gramian associated to the system (1.1) is the solution to

$$
A_{\theta} \Lambda_{\theta}+\Lambda_{\theta} A_{\theta}^{*}=-B_{\theta} B_{\theta}^{*}
$$

and is given by $\Lambda_{\theta}=\int_{0}^{\infty} e^{t A_{\theta}} B_{\theta} B_{\theta}^{*} e^{t A_{\theta}^{*}} \mathrm{~d} t$.

In order for the last integral to be convergent, one has to assume that $A_{\theta}$ is an exponentially stable operator for every $\theta \in \Theta$, thereby restricting the analysis to dissipative systems.

As for a surrogate distance of two Gramians associated to different parameters $\theta_{1}, \theta_{2}$, one attempts to follow the lines of $\S 3.1 .2$ and estimate it by a residual obtained by plugging one operator into the equation satisfied by the other one:

$$
c\left\|\Lambda_{\theta_{1}}-\Lambda_{\theta_{2}}\right\| \leqslant\left\|L_{A_{\theta_{1}}}\left(\Lambda_{\theta_{1}}-\Lambda_{\theta_{2}}\right)\right\| \leqslant C\left\|\Lambda_{\theta_{1}}-\Lambda_{\theta_{2}}\right\| .
$$

where $L_{A}$ stands for the Lyapunov operator given by $L_{A}(P)=A P+P A^{*}$.

The above inequalities are easy to obtain in case of finite dimensional systems (cf. [46]), while appropriate result in case of an unbounded operator $A_{\theta}$ requires subtle analysis and development of necessary estimates in appropriate functional spaces [29].

\section{Conclusion}

In this chapter we have presented three approaches for the computation of a control for parameter dependent system.

In the first approach, averaged controllability, we only build a control which steers the average of the system with respect to the parameter. This control is independent of the parameter and can be easily obtained. However, for a given realisation of the parameter, there is no guarantee that the system solution will be close to the target.

In the second approach, ensemble controllability, we build a single, parameter independent control such that all the realisations of the parameter dependent system approximately reach the target. This is the ideal situation. However, not all systems are ensemble controllable, and the numerical computation of the control involves to solve a system of high dimension which might be ill conditioned.

Finally, the last approach is to compute a control for each realisation of the parameter value. This approach is time consuming if we have to repeat the control construction for every realisation of the parameter. For this reason, we proposed a reduced basis approach, allowing a fast computation of an approximate control what ever the realisation of the parameter is. This approach implies an offline part consisting in building an adequate reduced basis.

For all these approaches, we have provided corresponding theoretical background (including convergence results), numerical algorithms and illustrative examples.

Some specific comments and open question are listed below.

Robust control. Roughly speaking, the aim of robust control is to build a feedback controller $K$, independent of the parameter, such that the origin is a stable point for the system $\dot{x}_{\theta}=\left(A_{\theta}+\right.$ $\left.B_{\theta} K\right) x_{\theta}$, for every realisation of the parameter $\theta$. This approach has been proposed in many text books and has not been developed in this chapter. We then refer the interested reader to books [2, 10, 15, 50].

Averaged controllability. In this chapter, we illustrated averaged controllability with an interpolation method based on the mid-point rule. However, if the set of parameters is a compact in $\mathbb{R}^{d}$, and if we have enough regularity with respect to the parameter, then any numerical integration 
will be fine. When the parameter set is unbounded, the major difficulty is to find or develop a convergent method for the numerical integration on this parameter set.

Ensemble controllability. In this chapter, we only consider the uniform ensemble controllability. As far as we know, there do not exist numerical approaches for finding a control for $L^{q}$-ensemble controllability (for $1 \leqslant q<\infty$ ), without assuming that the system is $L^{\infty}$-ensemble controllable.

Let us also point out that up to our best knowledge, there do not exist ensemble controllability results for PDE systems (with an infinite set of parameters). In regard to the sufficient conditions given in Proposition 2.14, checking the assumptions on the spectral map is a real challenge due to the infinite number of eigenvalues for PDE operators.

Reduced basis for solutions to differential Lyapunov equations. In Section 3.3 we have described the procedure of obtaining a greedy approximation to solutions of parameter dependent, algebraic Lyapunov equations. It would be interesting to develop a similar algorithm for their differential counterparts. As finite time Gramians are solutions to the latter, this would in turn allow application to control problems in finite time horizon, and this for general systems, either of dissipative nature or not. However, it remains to check the efficiency of such approach, as potential residuals are a priory time-dependent which might enlarge the computational cost and the required memory capacities.

Model order reduction. For all the methods proposed in this paper, the control is obtained by solving the primal and dual systems (of potentially large dimension). In particular, when applying a reduced basis approach, we repeat this in the offline phase for each value from the discretized parameter set, which makes its computational cost very high.

In order to bypass that problem, it would be useful to implement model reduction techniques which enable one to substitute a high dimensional system by an approximate one of a smaller dimension. Recent overviews of model order reduction methods for parametric (time) dependent problems is given in 7, 8, Chapter 9] (cf. also the other chapters of the same book). These have been successfully implemented for elliptic and parabolic optimal control problems [24, 23, but, as far as we know, they have not been developed in the context of controllability. Preliminary work in that direction is presented in [17, but rigorous error bounds still remain open.

Neural networks. A data-driven approach based on combination of reduced basis (RB) methods and machine learning has been the object of intense investigation during the last years. Although there is a vast literature on application of neural networks in mathematics, the available references significantly reduce when it comes to the issue of solving parameter dependent problems. In [21, 45, non-intrusive RB methods that rely on neural networks were successfully applied for computing solutions to parametric PDEs.

It would be interesting to develop a similar approach with the aim of efficiently treating parameter dependent control problems. The reduced basis algorithms were successfully developed in this context during the last decade, with the offline phase mainly exploring POD or a greedy sampling procedure. However, the cost of the corresponding online phase might still appear high, as the computation of projection (of solution to reduced basis) relies on the full-order model. An alternative approach would employ neural networks and train them to accurately predict the coefficients of solutions in reduced basis, with a computational cost independent of the dimension of the full-order model.

In addition, similar approach can be used in the context of simultaneous or ensemble controllability. Practical applications would include power and gas networks where recent attempts have been made at developing optimal control policies by using machine learning techniques in the offline phase 42 .

PDEs. In this chapter we have restricted the exposition to finite dimensional linear systems. However, it is important to stress that a number of positive control results has been obtained in the context of parameter dependent PDEs. This is in particular the case for averaged controllability 
and reduced basis approach. Note that all the methods proposed in this chapter will work well for discretized PDEs. However, some finer numerical analysis ensuring appropriate convergence results in that case is still missing. More precisely, one has to design the discretization of the PDE in terms of the discretization of the parameter set (or inversely), so that convergence of the method is ensured when both discretization constants go to 0 . This remains as a challenging open problem for future investigations.

\section{References}

[1] M. Abdelli and C. Castro. Numerical approximation of the averaged controllability for the wave equation with unknown velocity of propagation, 2020.

[2] J. Ackermann, A. Bartlett, D. Kaesbauer, W. Sienel, and R. Steinhauser. Robust control. Communications and Control Engineering Series. Springer-Verlag London, Ltd., London, 1993. Systems with uncertain physical parameters.

[3] A. Agrachev, Y. Baryshnikov, and A. Sarychev. Ensemble controllability by Lie algebraic methods. ESAIM Control Optim. Calc. Var., 22(4):921-938, 2016.

[4] J. Asier Bárcena-Petisco and E. Zuazua. Averaged dynamics and control for heat equations with random diffusion. working paper or preprint, Oct. 2020.

[5] A. S. Avetisyan and A. Z. Khurshudyan. Averaged controllability of Euler-Bernoulli beams with random material characteristics: the Green's function approach. Izv. Nats. Akad. Nauk Armen. Mekh., 72(4):7-18, 2019.

[6] K. Beauchard, J.-M. Coron, and P. Rouchon. Controllability issues for continuous-spectrum systems and ensemble controllability of Bloch equations. Comm. Math. Phys., 296(2):525-557, 2010 .

[7] P. Benner, S. Gugercin, and K. Willcox. A survey of projection-based model reduction methods for parametric dynamical systems. SIAM Review, 57(4):483531, 2015.

[8] P. Benner, M. Ohlberger, A. Cohen, and K. E. W. (Eds). Model Reduction and Approximation: Theory and Algorithms. SIAM, Philadelphia, 2017.

[9] J. M. Borwein and Q. J. Zhu. Techniques of variational analysis, volume 20 of CMS Books in Mathematics/Ouvrages de Mathématiques de la SMC. Springer-Verlag, New York, 2005.

[10] X.-H. Chang. Robust output feedback H-infinity control and filtering for uncertain linear systems, volume 7 of Studies in Systems, Decision and Control. Springer, Heidelberg, 2014.

[11] X. Chen. Structure theory for ensemble controllability, observability, and duality. Math. Control Signals Systems, 31(2):Art. 7, 40, 2019.

[12] J. Coulson, B. Gharesifard, and A.-R. Mansouri. On average controllability of random heat equations with arbitrarily distributed diffusivity. Automatica J. IFAC, 103:46-52, 2019.

[13] R. DeVore, G. Petrova, and P. Wojtasczyk. Greedy algorithms for reduced bases in banach spaces. Constructive Approximation, 37(1):455-466, 2013.

[14] G. Dirr and M. Schnlein. Uniform and $L^{q}$-ensemble reachability of parameter-dependent linear systems, 2020. 
[15] P. Dorato, L. Fortuna, and G. Muscato. Robust control for unstructured perturbations - an introduction, volume 168 of Lecture Notes in Control and Information Sciences. SpringerVerlag, Berlin, 1992.

[16] I. Ekeland and R. Témam. Convex analysis and variational problems, volume 28 of Classics in Applied Mathematics. Society for Industrial and Applied Mathematics (SIAM), Philadelphia, PA, english edition, 1999. Translated from the French.

[17] G.Fabrini, L. Iapichino, and S.Volkwein. Reduced-order greedy controllability of finite dimensional linear systems. IFAC-PapersOnLine, 51(2):296-301, 2018.

[18] B. Haasdonk. Convergence rates of the podgreedy method. ESAIM: M2AN, 47(3):859 - 873, 2013.

[19] U. Helmke and M. Schönlein. Uniform ensemble controllability for one-parameter families of time-invariant linear systems. Systems Control Lett., 71:69-77, 2014.

[20] V. Hernndez-Santamara, M. Lazar, and E. Zuazua. Greedy optimal control for elliptic problems and its application to turnpike problems. Numerische Mathematik, 141(1):455-493, 2019.

[21] J. Hesthaven and S. Ubbiali. Non-intrusive reduced order modeling of nonlinear problems using neural network. J. Comput. Phys., 363:55-78, 2018.

[22] J. S. Hesthaven, G. Rozza, and B. Stamm. Certified reduced basis methods for parametrized partial differential equations. Springer International Publishing, Berlin, 2016.

[23] M. Kärcher and M. A. Grepl. A posteriori error estimation for reduced order solutions of parametrized parabolic optimal control problems. ESAIM Math. Model. Numer. Anal., 48(6):1615-1638, 2014.

[24] M. Kärcher and M. A. Grepl. A certified reduced basis method for parametrized elliptic optimal control problems. ESAIM Control Optim. Calc. Var., 20(2):416-441, 2014.

[25] J. Klamka and A. Z. Khurshudyan. Averaged controllability of heat equation in unbounded domains with random geometry and location of controls: the Green's function approach. Arch. Control Sci., 29(65)(4):573-584, 2019.

[26] E. Kreindler and P. E. Sarachik. On the concepts of controllability and observability of linear systems. IEEE Trans. Automatic Control, AC-9:129-136, 1964.

[27] M. Lazar. Stability of observations of partial differential equations under uncertain perturbations. ESAIM Control Optim. Calc. Var., 24(1):45-61, 2018.

[28] M. Lazar and J. Lohéac. Output controllability in a long-time horizon. Automatica J. IFAC, $113: 108762,8,2020$.

[29] M. Lazar and J. Weston. Greedy algorithm for parameter dependent operator Lyapunov equations. preprint, 2020.

[30] M. Lazar and E. Zuazua. Averaged control and observation of parameter-depending wave equations. C. R. Math. Acad. Sci. Paris, 352(6):497-502, 2014.

[31] M. Lazar and E. Zuazua. Greedy controllability of finite dimensional linear systems. Automatica, 14(1):327-340, 2016. 
[32] J.-S. Li. Ensemble control of finite-dimensional time-varying linear systems. IEEE Trans. Automat. Control, 56(2):345-357, 2011.

[33] J.-S. Li and N. Khaneja. Ensemble control of Bloch equations. IEEE Trans. Automat. Control, $54(3): 528-536,2009$.

[34] J.-S. Li and J. Qi. Ensemble control of time-invariant linear systems with linear parameter variation. IEEE Trans. Automat. Control, 61(10):2808-2820, 2016.

[35] J.-S. Li, W. Zhang, and L. Tie. On Separating Points for Ensemble Controllability. SIAM J. Control Optim., 58(5):2740-2764, 2020.

[36] J.-L. Lions. Contrôlabilité exacte, perturbations et stabilisation de systèmes distribués. Tome 1 , volume 8 of Recherches en Mathématiques Appliquées [Research in Applied Mathematics]. Masson, Paris, 1988. Contrôlabilité exacte. [Exact controllability], With appendices by E. Zuazua, C. Bardos, G. Lebeau and J. Rauch.

[37] J. Lohéac and E. Zuazua. From averaged to simultaneous controllability. Ann. Fac. Sci. Toulouse Math. (6), 25(4):785-828, 2016.

[38] J. Lohéac and E. Zuazua. Averaged controllability of parameter dependent conservative semigroups. J. Differential Equations, 262(3):1540-1574, 2017.

[39] Q. Lü and E. Zuazua. Averaged controllability for random evolution partial differential equations. J. Math. Pures Appl. (9), 105(3):367-414, 2016.

[40] Y. Maday. Reduced basis method for the rapid and reliable solution of partial differential equations. In International Congress of Mathematicians, 2006., pages 1255-1270. European Mathematical Society, 2006.

[41] F. J. Marín, J. Martínez-Frutos, and F. Periago. Robust averaged control of vibrations for the Bernoulli-Euler beam equation. J. Optim. Theory Appl., 174(2):428-454, 2017.

[42] Y. Ng, S. Misra, L. Roald, and S. Backhaus. Statistical learning for dc optimal power flow. In Power Systems Computation Conference (PSCC), IEEE, pages 1-7, 2018.

[43] A. Quarteroni, A. Manzoni, and F. Negri. Reduced basis methods for partial differential equations, volume 92 of Unitext. Springer, Cham, 2016. An introduction, La Matematica per il $3+2$.

[44] D. L. Russell. The Dirichlet-Neumann boundary control problem associated with Maxwell's equations in a cylindrical region. SIAM J. Control Optim., 24(2):199-229, 1986.

[45] N. D. Santo, S. Deparis, and L. Pegolotti. Data driven approximation of parametrized pdes by reduced basis and neural networks. J. Comput. Phys., 416:109550, 2020.

[46] N. Son and T.Stykel. Solving parameter-dependent Lyapunov equations using the reduced basis method with application to parametric model order reduction. SIAM Journal on Matrix Analysis and Applications, 38(2):478-504, 2017.

[47] R. Triggiani. Controllability and observability in Banach space with bounded operators. SIAM J. Control, 13:462-491, 1975.

[48] M. Tucsnak and G. Weiss. Observation and Control for Operator Semigroups. BirkhauserVerlag AG, Berlin, 2009. 
[49] K. Veroy, C. PrudHomme, D. Rovas, and A. Patera. A posteriori error bounds for reducedbasis approximation of parametrized noncoercive and nonlinear elliptic partial differential equations. In 16th AIAA Computational Fluid Dynamics Conference. Orlando, United States., 2003.

[50] R. K. Yedavalli. Robust control of uncertain dynamic systems. Springer, New York, 2014. A linear state space approach.

[51] S. Zeng and F. Allgöwer. A moment-based approach to ensemble controllability of linear systems. Systems Control Lett., 98:49-56, 2016.

[52] E. Zuazua. Controllability and observability of partial differential equations: Some results and open problems. In Handbook of Differential Equations: Evolutionary Equations, vol. 3, C. M. Dafermos and E. Feireisl (eds),, pages 527-621. Elsevier Science, Amsterdam., 2006.

[53] E. Zuazua. Averaged control. Automatica J. IFAC, 50(12):3077-3087, 2014. 